

\title{
Dual graded graphs for Kac-Moody algebras
}

\author{
Thomas F. Lam and Mark Shimozono
}

\begin{abstract}
Motivated by affine Schubert calculus, we construct a family of dual graded graphs $\left(\Gamma_{s}, \Gamma_{w}\right)$ for an arbitrary Kac-Moody algebra $\mathfrak{g}$. The graded graphs have the Weyl group $W$ of $\mathfrak{g e h}$ as vertex set and are labeled versions of the strong and weak orders of $W$ respectively. Using a construction of Lusztig for quivers with an admissible automorphism, we define folded insertion for a Kac-Moody algebra and obtain Sagan-Worley shifted insertion from Robinson-Schensted insertion as a special case. Drawing on work of Proctor and Stembridge, we analyze the induced subgraphs of $\left(\Gamma_{s}, \Gamma_{w}\right)$ which are distributive posets.
\end{abstract}

\section{Introduction}

The Robinson-Schensted correspondence is perhaps the most important algorithm in algebraic combinatorics. It exhibits a bijection between permutations and pairs of standard Young tableaux of the same shape. Stanley [1988] investigated the class of differential posets (also studied in [Fomin 1986]). Fomin [1994] studied the more general notion of a dual graded graph to formalize local conditions which guarantee the existence of a Robinson-Schensted style algorithm.

In this article, we construct a family of dual graded graphs $\left(\Gamma_{s}, \Gamma_{w}\right)$ associated to each Kac-Moody algebra $\mathfrak{g}$. These graded graphs have as vertex set the Weyl group $W$ of $\mathfrak{g}$. The pair $\left(\Gamma_{s}, \Gamma_{w}\right)=\left(\Gamma_{s}(\Lambda), \Gamma_{w}(K)\right)$ depends on a pair $(\Lambda, K)$ where $\Lambda$ is a dominant integral weight and $K$ is a "positive integral" element of the center $Z(\mathfrak{g})$. In every case $\Gamma_{w}$ is obtained by labeling the left weak order of $W$ and $\Gamma_{s}$ is obtained by labeling the strong Bruhat order of $W$.

These labelings are motivated by the Schubert calculus for homogeneous spaces associated to the Kac-Moody group $G$ with Lie algebra $\mathfrak{g}$. For $w \in W$, let $\xi^{w} \in$ $H^{*}(G / B)$ denote the cohomology Schubert classes of the flag manifold of $G$. If $\Lambda=\Lambda_{i}$ is the $i$-th fundamental weight, then an edge $v \lessdot w$ in $\Gamma_{s}(\Lambda)$ is labeled with

MSC2000: primary 05E10; secondary 57T15, $17 \mathrm{~B} 67$.

Keywords: dual graded graphs, Schensted insertion, affine insertion.

Lam was partially supported by NSF DMS-0600677. Shimozono was partially supported by NSF DMS-0401012. 
the coefficient of $\xi^{w}$ in the product $\xi^{s_{i}} \xi^{v}$, also called a Chevalley coefficient. When $\mathfrak{g}$ is of affine type and $K=K_{\text {can }}$ is the canonical central element, the analogous statement holds (Proposition 2.17) for $\Gamma_{w}(K)$ with the homology Schubert classes $\xi_{w} \in H_{*}(\mathrm{Gr})$ of the affine Grassmannian corresponding to $\mathfrak{g}$ replacing the cohomology classes. Thus the combinatorics of these graphs encode computations in Schubert calculus, and the duality of the graded graphs $\left(\Gamma_{s}, \Gamma_{w}\right)$ is a combinatorial skeleton of the duality between cohomology and homology of homogeneous spaces of $G$.

In the case of the affine Grassmannian, the dual graded graph structure arises from the pair of dual graded Hopf algebras given by $H_{*}(\mathrm{Gr})$ and $H^{*}(\mathrm{Gr})$ : one may define the down operator by the action of the homology class $\xi_{s_{0}}$ on the Schubert basis of $H^{*}(\mathrm{Gr})$ and the up operator by multiplication by $\xi^{s_{i}}$ for any fixed simple reflection $s_{i}$. It is a general phenomenon that pairs of dual graded combinatorial Hopf algebras yield dual graded graphs; we shall pursue this in a separate publication [Lam and Shimozono $\geq 2007$ ].

Chains in the graded graphs $\left(\Gamma_{s}, \Gamma_{w}\right)$, which we call strong and weak tableaux, are natural generalizations of standard Young tableaux. To go one speculative step further, we believe that the generating functions of an appropriate semistandard notion of strong and weak tableaux would give polynomials which represent certain homology and cohomology Schubert classes, in particular for homogeneous spaces corresponding to maximal parabolics, generalizing Schur functions, Schur $Q$-functions and the like. While this statement is vague in general, it can be made much more precise when $\mathfrak{g}$ is of affine type, and has already been achieved in one case.

In the case that $\mathfrak{g}$ is of the affine type $A_{n-1}^{(1)}$ our construction recovers the dual graded graphs that were implicitly studied in our joint work with Lapointe and Morse [2006]. The weak and strong tableaux in [Lam et al. 2006] are semistandard generalizations of the corresponding objects here; in the same work, an affine insertion algorithm was explicitly constructed for semistandard weak and strong tableaux, and from [Lam 2006; Lam et al. 2006] we know that the corresponding generating functions do indeed represent Schubert classes of the affine Grassmannian of type $A$. In the limit $n \rightarrow \infty$ of the $A_{n-1}^{(1)}$ case, our construction reproduces Young's lattice, which is the self-dual graded graph that gives rise to the RobinsonSchensted algorithm.

Having constructed the Kac-Moody dual graded graphs we study two further aspects of these graphs in detail.

The first aspect is motivated by the relation between the Robinson-Schensted insertion and Sagan-Worley shifted insertion. Using Lusztig's construction [1993] which associates to each symmetrizable generalized Cartan matrix $A$, a symmetric generalized Cartan matrix $B$ equipped with an admissible automorphism $\pi$, we 
show that any dual graded graph of the form $\left(\Gamma_{s}^{A}, \Gamma_{w}^{A}\right)$ for $\mathfrak{g}(A)$ can be realized in terms of one of the form $\left(\Gamma_{s}^{B}, \Gamma_{w}^{B}\right)$ for $\mathfrak{g}(B)$. In particular, for any affine algebra, any of the dual graded graphs $\left(\Gamma_{s}, \Gamma_{w}\right)$ can be realized using a dual graded graph for a simply-laced affine algebra. In particular we obtain a Schensted bijection for type $C_{n}^{(1)}$, using the insertion algorithm of [Lam et al. 2006] for type $A_{2 n-1}^{(1)}$. As $n$ goes to infinity, the type $C_{n}^{(1)}$ insertion converges to Sagan-Worley insertion [Sagan 1987; Worley 1984]. As a related result, we define a notion of mixed insertion for dual graded graphs equipped with a pair of automorphisms. This generalizes Haiman's variants of Schensted insertion known as left-right, mixed, and doubly dual insertion [Haiman 1989].

The second aspect we investigate are the induced subgraphs of the pair $\left(\Gamma_{s}, \Gamma_{w}\right)$ which are distributive lattices when considered as posets. These are precisely the conditions under which one may describe our strong and weak tableaux by "filling cells with numbers" as in a usual standard Young tableau. Here we draw on [Proctor 1984; 1999; Stembridge 1996], which classify the parabolic quotients of Weyl groups of simple Lie algebras whose left weak orders (or equivalently Bruhat orders) are distributive lattices. We sharpen these results slightly to show that in these cases, the distributivity is compatible with the edge labels of the graphs $\left(\Gamma_{s}, \Gamma_{w}\right)$; see Section 6B. These distributive parabolic quotients have also appeared recently in the geometric work [Thomas and Yong 2006]. They show that in these cases one may use the jeu-de-taquin to calculate Schubert structure constants of the cohomology of (co)minuscule flag varieties. We do not recover this result, but we note that their notion of standard tableau, fits into our framework as strong (or weak) tableaux for the distributive parabolic quotients, with the edge labels forgotten.

\section{Dual graded graphs for Kac-Moody algebras}

2A. Dual graded graphs. We recall Fomin's notion of dual graded graphs [Fomin 1994]. A graded graph is a directed graph

$$
\Gamma=(V, E, h, m)
$$

with vertex set $V$ and set of directed edges $E \subset V^{2}$, together with a grading function $h: V \rightarrow \mathbb{Z}_{\geq 0}$, such that every directed edge $(v, w) \in E$ satisfies $h(w)=h(v)+1$ and has a multiplicity $m(v, w) \in \mathbb{Z}_{\geq 0}$. Forgetting the edge labels $m, \Gamma$ may be regarded as the Hasse diagram of a graded poset. We shall interpret $m(v, w)$ as making $\Gamma$ into a directed multigraph in which there are $m(v, w)$ distinct edges from $v$ to $w$.

$\Gamma$ is locally finite if, for every $v \in V$, there are finitely many $w \in V$ such that $(v, w) \in E$ and finitely many $u \in V$ such that $(u, v) \in E$; we shall assume this condition without further mention. For a graded graph $\Gamma=(V, E, h, m)$ define the 
$\mathbb{Z}$-linear down and up operators $D, U: \mathbb{Z} V \rightarrow \mathbb{Z} V$ on the free abelian group $\mathbb{Z} V$ of formal $\mathbb{Z}$-linear combinations of vertices, by

$$
U_{\Gamma}(v)=\sum_{(v, w) \in E} m(v, w) w \quad \text { and } \quad D_{\Gamma}(w)=\sum_{(v, w) \in E} m(v, w) v .
$$

A pair of graded graphs $\left(\Gamma, \Gamma^{\prime}\right)$ is $d u a l$ if $\Gamma$ and $\Gamma^{\prime}$ have the same vertex sets and grading function but possibly different edge sets and edge multiplicities, such that

$$
D_{\Gamma^{\prime}} U_{\Gamma}-U_{\Gamma} D_{\Gamma^{\prime}}=r \mathrm{Id}
$$

as $\mathbb{Z}$-linear operators on $\mathbb{Z} V$, for some fixed $r \in \mathbb{Z}_{>0}$. We call $r$ the differential coefficient. When $\Gamma=\Gamma^{\prime}$ and all the edges have multiplicity one, we obtain the $r$-differential posets of [Stanley 1988].

Remark 2.1. The duality property implies that $V$ is infinite.

Example 2.2. Let $\Gamma=\mathbb{Y}$ be Young's lattice, with $(\lambda, \mu) \in E$ if the diagram of the partition $\mu$ is obtained from that of $\lambda$ by adding a single cell (in which case we say that the cell is $\lambda$-addable and $\mu$-removable), all edge multiplicities are 1 , and $h(\lambda)=|\lambda|$ is the number of cells in the diagram of $\lambda$. Then $(\mathbb{Y}, \mathbb{Y})$ is a pair of dual graded graphs with differential coefficient 1 .

2B. The labeled Kac-Moody weak and strong orders. In this section a new family of dual graded graphs is introduced.

Let $I$ be a set of Dynkin nodes and $A=\left(a_{i j}\right)_{i, j \in I}$ be a generalized Cartan matrix (GCM), that is, one with integer entries which satisfies $a_{i i}=2$ for all $i \in I$, and for all $i \neq j, a_{i j} \leq 0$ and $a_{i j}<0$ if and only if $a_{j i}<0$. Let $\mathfrak{g}=\mathfrak{g}(A)$ denote the KacMoody algebra over $\mathbb{C}$ associated to $A$ [Kac 1990], $\mathfrak{h} \subset \mathfrak{g}$ the Cartan subalgebra, and $\mathfrak{h}^{*}$ the dual. Let $\left\{\alpha_{i} \mid i \in I\right\} \subset \mathfrak{h}^{*}$ be the simple roots, $\left\{\alpha_{i}^{\vee} \mid i \in I\right\} \subset \mathfrak{h}$ the simple coroots, and $\left\{\Lambda_{i} \mid i \in I\right\} \subset \mathfrak{h}^{*}$ the fundamental weights, with $a_{i j}=\left\langle\alpha_{i}^{\vee}, \alpha_{j}\right\rangle$ where $\langle\cdot, \cdot\rangle: \mathfrak{h} \times \mathfrak{h}^{*} \rightarrow \mathbb{C}$ is the natural pairing. We assume that the simple roots are linearly independent and the dimension of $\mathfrak{h}$ is chosen to be minimal. Let $W$ be the Weyl group of $\mathfrak{g}$ : it has generators $s_{i}$ for $i \in I$ and relations $s_{i}^{2}=1$ for $i \in I$ and $\left(s_{i} s_{j}\right)^{m_{i j}}=1$ for $i, j \in I$ with $i \neq j$, where $m_{i j}$ is $2,3,4,6$ or $\infty$ according as $a_{i j} a_{j i}$ is $0,1,2,3$ or $>3$. Let $\ell: W \rightarrow \mathbb{Z}_{\geq 0}$ be the length function on $W$. Let $\Delta_{\mathrm{re}}=W \cdot\left\{\alpha_{i} \mid i \in I\right\}$ be the set of real roots and $\Delta_{\mathrm{re}}^{+}=\Delta_{\mathrm{re}} \cap \bigoplus_{i \in I} \mathbb{Z}_{\geq 0} \alpha_{i}$ the positive real roots. The associated coroot $\alpha^{\vee}$ of $\alpha \in \Delta_{\text {re }}^{+}$is defined by $\alpha^{\vee}=u \alpha_{i}^{\vee}$, where $u \in W$ and $i \in I$ are such that $\alpha=u \alpha_{i}$. For $\alpha \in \Delta_{\text {re }}$ let $s_{\alpha}=u s_{i} u^{-1}$ denote the reflection associated to $\alpha$. The strong order (or Bruhat order) $\leq$ on $W$ is defined by the cover relations $w \lessdot w s_{\alpha}$ whenever $\ell\left(w s_{\alpha}\right)=\ell(w)+1$ for some $\alpha \in \Delta_{\text {re }}^{+}$and $w \in W$. The left weak order $(W, \preceq)$ is the subposet of $(W, \leq)$ generated by the cover relations $w \prec s_{i} w$ whenever $\ell\left(s_{i} w\right)=\ell(w)+1$ for some $i \in I$ and $w \in W$. The left descent set of $v$ is defined by $\operatorname{Des}(v)=\left\{i \in I \mid s_{i} v \prec v\right\}$. 
Given $\Lambda$ in the set $P^{+}$of dominant integral weights, let $\Gamma_{s}(\Lambda)$ be the graded graph with vertex set $W$ and edges $(v, w) \in W^{2}$ such that $v \lessdot w$, with multiplicity $m_{\Lambda}(v, w)=\left\langle\alpha^{\vee}, \Lambda\right\rangle$, where $\alpha \in \Delta_{\text {re }}^{+}$is such that $w=v s_{\alpha}$. Let $i \in I$ and $u \in W$ be such that $\alpha=u \alpha_{i}$. Then

$$
m_{\Lambda}(v, w)=\left\langle u \alpha_{i}^{\vee}, \Lambda\right\rangle=\left\langle\alpha_{i}^{\vee}, u^{-1} \Lambda\right\rangle .
$$

Let $Z^{+}=Z^{+}(\mathfrak{g}(A))=Z(\mathfrak{g}(A)) \cap \bigoplus_{i \in I} \mathbb{Z}_{\geq 0} \alpha_{i}^{\vee}$, where $Z(\mathfrak{g}(A))$ is the center of $\mathfrak{g}(A)$. If $K \in Z^{+}$, writing $K=\sum_{i \in I} k_{i} \alpha_{i}^{\vee}$, the vector $\left(k_{i}\right)_{i \in I}$ defines a linear dependence amongst the rows of $A$.

Given $K \in Z^{+}$, let $\Gamma_{w}(K)$ be the graded graph with vertex set $W$ and edges $(v, w) \in W^{2}$ such that $v \prec w=s_{i} v$, with multiplicity

$$
n_{K}(v, w)=k_{i}=\left\langle K, \Lambda_{i}\right\rangle .
$$

Both $\Gamma_{s}(\Lambda)$ and $\Gamma_{w}(K)$ are graded by the length function.

Theorem 2.3. Let $(\Lambda, K) \in P^{+} \times Z^{+}$. Then $\left(\Gamma_{s}(\Lambda), \Gamma_{w}(K)\right)$ is a pair of dual graded graphs with differential coefficient $r=\langle K, \Lambda\rangle$.

Proof. Let $U=U_{\Gamma_{s}(\Lambda)}$ and $D=D_{\Gamma_{w}(K)}$. The coefficient of $u \neq v$ in $(D U-U D) v$ is given by

$$
\sum_{\substack{i, \alpha) \in I \times \Delta_{\mathrm{re}}^{+} \\ v<v s_{S^{\prime}} \\ u=s_{i} v s_{\alpha} \prec v s_{\alpha}}} k_{i}\left\langle\alpha^{\vee}, \Lambda\right\rangle-\sum_{\substack{(i, \alpha) \in I \times \Delta_{\mathrm{re}}^{+} \\ s_{i} v<\Delta_{i} v<s_{i} v s_{\alpha}=u}} k_{i}\left\langle\alpha^{\vee}, \Lambda\right\rangle .
$$

This quantity is zero because the indexing sets of both sums coincide, by two versions of [Humphreys 1990, Lemma 5.11].

For every $i \in I$ and $v \in W$, either $v \prec s_{i} v$ or $s_{i} v \prec v$ is a covering relation. It follows that the coefficient of $v$ in $(D U-U D) v$ is

$$
\begin{aligned}
\sum_{i \in I \backslash \operatorname{Des}(v)} k_{i}\left\langle v^{-1} \alpha_{i}^{\vee}, \Lambda\right\rangle-\sum_{i \in \operatorname{Des}(v)} k_{i}\left\langle\left(s_{i} v\right)^{-1} \alpha_{i}^{\vee}, \Lambda\right\rangle & \\
& =\sum_{i \in I} k_{i}\left\langle v^{-1} \alpha_{i}^{\vee}, \Lambda\right\rangle=\sum_{i \in I} k_{i}\left\langle\alpha_{i}^{\vee}, v \Lambda\right\rangle \\
& =\langle K, v \Lambda\rangle=\left\langle v^{-1} K, \Lambda\right\rangle=\langle K, \Lambda\rangle .
\end{aligned}
$$

We have used the $W$-invariance of $\langle\cdot, \cdot\rangle$ and $K$.

Remark 2.4. For $i \in I$ and $v \in W$, the multiplicity of the edge $\left(v, v s_{\alpha}\right)$ in $\Gamma_{s}\left(\Lambda_{i}\right)$, is the Chevalley multiplicity, given by the coefficient of $\xi^{v s_{\alpha}}$ in the product $\xi^{s_{i}} \xi^{v}$, where $\xi^{v} \in H^{*}(G / B)$ is the Schubert cohomology class for the flag manifold $G / B$ associated with the Kac-Moody algebra $\mathfrak{g}$ [Kostant and Kumar 1986].

In Proposition 2.17 we will relate the multiplicities of the weak graph $\Gamma_{w}(K)$ with the homology multiplication of the affine Grassmannian, in the case that $\mathfrak{g}(A)$ is of untwisted affine type. 
2C. Tableaux and enumeration. Let $\Gamma=(V, E, h, m)$ be a graded graph and $v, w \in V$. А $\Gamma$-tableau $T$ of shape $v / w$ is a directed path

$$
T=\left(w=v_{0} \stackrel{m_{1}}{\longrightarrow} v_{1} \stackrel{m_{2}}{\longrightarrow} \cdots \stackrel{m_{k}}{\longrightarrow} v_{k}=v\right)
$$

from $w$ to $v$ in $\Gamma$, where $m_{i}$ is an element taken from a set of $m\left(v_{i-1}, v_{i}\right)$ possible markings of the edge $\left(v_{i-1}, v_{i}\right)$. In the multigraph interpretation, $m_{i}$ indicates which of the $m\left(v_{i-1}, v_{i}\right)$ edges going from $v_{i-1}$ to $v_{i}$, is traversed by the path.

Let $v=\operatorname{out}(T)$ and $w=\operatorname{in}(T)$ the outer and inner shapes of $T$ respectively, and denote by $\mathscr{T}(\Gamma)$ the set of $\Gamma$-tableaux, and $\mathscr{T}(\Gamma, v / w)$ the subset of those of shape $v / w$.

Example 2.5. For $\mathbb{Y}$ as in Example 2.2, $צ$-tableaux are standard Young tableaux.

If $\Gamma$ has a unique minimum element $\hat{0}$, we say $T$ has shape $v$ if in $(T)=\hat{0}$.

Theorem 2.6 [Fomin 1994]. Let $\left(\Gamma, \Gamma^{\prime}\right)$ be a pair of dual graded graphs with differential coefficient $r$. Then

$$
r^{n} n !=\sum_{\substack{v \in V \\ h(v)=n}} f_{\Gamma}^{v} f_{\Gamma^{\prime}}^{v}
$$

where $f_{\Gamma}^{v}=|\mathscr{T}(\Gamma, v)|$ and $f_{\Gamma^{\prime}}^{v}=\left|\mathscr{T}\left(\Gamma^{\prime}, v\right)\right|$.

Example 2.7. Let $\Gamma=\Gamma^{\prime}=\mathbb{Y}$. Then by Examples 2.2 and 2.5, Equation (4) is the well known identity $n !=\sum_{\lambda} f_{\lambda}^{2}$, where $\lambda$ ranges over the partitions of $n$ and $f_{\lambda}$ is the number of standard Young tableaux of shape $\lambda$.

The graphs $\Gamma_{w}(K)$ and $\Gamma_{s}(\Lambda)$ both have minimum element id $\in W$. We call $\Gamma_{w}(K)$-tableaux (standard) $K$-weak tableaux and $\Gamma_{s}(\Lambda)$-tableaux (standard) $\Lambda$ strong tableaux.

Corollary 2.8. Let $\mathfrak{g}$ be a Kac-Moody algebra and $(\Lambda, K) \in P^{+} \times Z^{+}$. Then for each $n \in \mathbb{Z}_{\geq 0}$ we have

$$
r^{n} n !=\sum_{\substack{w \in W \\ \ell(w)=n}} f_{\text {weak }}^{w} f_{\text {strong }}^{w}
$$

where $r=\langle K, \Lambda\rangle, f_{\text {weak }}^{w}$ is the number of $K$-weak tableaux of shape $w$ and $f_{\text {strong }}^{w}$ is the number of $\Lambda$-strong tableaux of shape $w$.

In Section 3 standard Young tableaux are realized as special cases of both $K$ weak and $\Lambda$-strong tableaux using affine algebras of type $A^{(1)}$. 
2D. From dual graded graphs to Schensted bijections. A differential bijection for the pair of dual graded graphs $\left(\Gamma, \Gamma^{\prime}\right)$ is one that exhibits the equality (1); we give a precise definition below. By [Fomin 1995], a differential bijection induces a Schensted bijection (see (8)) which describes the enumerative identity (4).

We recall Fomin's theory in more detail. Given $r \in \mathbb{Z}_{\geq 0}$, let $S(r)$ be a set of cardinality $r$. Sometimes we may write $S(r)$ for a particular set of cardinality $r$. Let $\left(\Gamma, \Gamma^{\prime}\right)$ be a pair of dual graded graphs with $\Gamma=(V, E, h, m)$ and $\Gamma^{\prime}=\left(V, E^{\prime}, h, m^{\prime}\right)$ and differential coefficient $r$. Given $x, y \in V$, define $\mathcal{U D}_{x y}=$ $\left\{\left(z, m, m^{\prime}\right) \in V \times \mathbb{Z}_{>0}^{2} \mid(z, y) \in E,(z, x) \in E^{\prime}, m \leq m(z, y), m^{\prime} \leq m^{\prime}(z, x)\right\}$ and let DU $u_{x y}=\left\{\left(w, M, M^{\prime}\right) \in V \times \mathbb{Z}_{>0}^{2} \mid(x, w) \in E,(y, w) \in E^{\prime}, M \leq m(x, w), M^{\prime} \leq\right.$ $\left.m^{\prime}(y, w)\right\} . \mathscr{U}_{x y}$ represents the set of marked paths going down one step in $\Gamma^{\prime}$ from $x$ to some $z \in V$ and then up one step in $\Gamma$ from $z$ to $y$. Similarly, $\mathscr{D} u_{x y}$ represents the set of marked paths going up one step in $\Gamma$ from $x$ to some $w \in V$, and then down one step in $\Gamma^{\prime}$ from $w$ to $y$. To cancel the off-diagonal terms in (1), for every $(x, y) \in V^{2}$ with $x \neq y$, there must be a bijection

$$
\Phi_{x y}: \mathscr{U D}_{x y} \rightarrow \mathscr{D} \mathcal{U}_{x y}
$$

and to obtain agreement of diagonal terms in (1), for each $x \in V$ there must be a bijection

$$
\Phi_{x}: S(r) \sqcup \cup \mathscr{D}_{x} \rightarrow \mathscr{D}_{x}
$$

where $\mathscr{D} u_{x}=\mathscr{D} \boldsymbol{U}_{x x}$ and $\mathfrak{U D}_{x}=\mathfrak{U D}_{x x}$. By definition, a differential bijection for $\left(\Gamma, \Gamma^{\prime}\right)$, is a collection $\Phi=\left(\Phi_{x y} ; \Phi_{x}\right)$ of such bijections $\Phi_{x y}$ and $\Phi_{x}$.

Example 2.9. Let $\Gamma=\mathbb{Y}$ with dual graded graph structure on $(\mathbb{Y}, \mathbb{Y})$ as in Example 2.2. For $\lambda \in \mathbb{Y}$, since all edges have multiplicity 1 , $\mathscr{D} u_{\lambda}$ is in bijection with $\lambda$ addable corner cells and $U_{\lambda}$ is in bijection with $\lambda$-removable corner cells. The $\lambda$ addable and $\lambda$-removable corner cells of $\lambda$ are interleaved. Let $\Phi_{\lambda}$ send the unique element of the set $S(1)$, to the $\lambda$-addable corner cell in the first row of $\lambda$, and send a $\lambda$-removable corner to the nearest $\lambda$-addable corner with higher row index. For $\lambda \neq \mu$ the sets $\mathscr{D} u_{\lambda \mu}$ and $\mathcal{D}_{\lambda \mu}$ have the same cardinality, which is either 0 or 1 , so there is no choice for the definition of $\Phi_{\lambda \mu}$. This defines a differential bijection $\Phi$ for $(\mathbb{Y}, \mathbb{Y})$.

Let $P_{n}(r)$ be the set of $r$-colored permutations of $n$ elements. We realize $\sigma \in$ $P_{n}(r)$ as an $n \times n$ monomial matrix (one with exactly one nonzero element in each row and in each column, whose nonzero entries must be taken from a set $S(r)$ of cardinality $r$ such that $0 \notin S(r))$.

We assume that $\Gamma$ and $\Gamma^{\prime}$ have a common minimum element $\hat{0}$ such that $h(\hat{0})=0$. 
A Schensted bijection I for $\left(\Gamma, \Gamma^{\prime}\right)$ is a family of bijections (for all $n \in \mathbb{Z}_{\geq 0}$ )

$$
\begin{aligned}
P_{n}(r) & \rightarrow \bigsqcup_{\substack{v \in V \\
h(v)=n}} \mathscr{T}(\Gamma, v) \times \mathscr{T}\left(\Gamma^{\prime}, v\right) \\
\sigma & \mapsto(P, Q) .
\end{aligned}
$$

We fix a differential bijection $\Phi$ for $\left(\Gamma, \Gamma^{\prime}\right)$ and define the induced Schensted bijection $\mathrm{I}_{\Phi}$.

Given $\sigma \in P_{n}(r)$, we shall define a directed graph $G$ with vertices $G_{i j} \in V$ for $0 \leq i, j \leq n$, which is depicted matrix-style. It shall have the property that (1) adjacent vertices $G_{i, j-1}, G_{i j}$ in a row, are either equal, or they form an edge $\left(G_{i, j-1}, G_{i j}\right) \in E$ with marking $m \in S\left(m\left(G_{i, j-1}, G_{i j}\right)\right)$, and (2) adjacent vertices $G_{i-1, j}, G_{i j}$ in a column, are either equal, or they form an edge $\left(G_{i-1, j}, G_{i j}\right) \in$ $E^{\prime}$ with marking $m^{\prime} \in S\left(m^{\prime}\left(G_{i-1, j}, G_{i j}\right)\right)$. Moreover, (3) $G_{i-1, j} \neq G_{i j}$ (resp. $G_{i, j-1} \neq G_{i j}$ ) if and only if the unique $p$ such that $\sigma_{p j} \neq 0$ (resp. $q$ such that $\sigma_{i q} \neq 0$ ) satisfies $p \leq i$ (resp. $q \leq j$ ). In particular (ignoring the equalities), for each $i$, the $i$-th row $G_{i \bullet}$ is a $\Gamma$-tableau and for each $j$, the $j$-th column $G_{\bullet j}$ is a $\Gamma^{\prime}$-tableau. For the sake of uniform language we shall always imagine that there is a marked edge $G_{i, j-1} \rightarrow G_{i j}$ and $G_{i-1, j} \rightarrow G_{i j}$, but when the vertices coincide the marked edge degenerates.

$G$ is defined inductively as follows. The north and west edges $G_{0 \bullet}$ and $G_{\bullet 0}$ of $G$ are initialized to the empty tableau: $G_{i 0}=G_{0 j}=\hat{0}$ for all $0 \leq i, j \leq n$. To define the rest of $G$, it suffices to give a local rule, which, given the marked edges

$$
G_{i-1, j-1} \stackrel{m}{\rightarrow} G_{i-1, j} \quad \text { and } \quad G_{i-1, j-1} \stackrel{m^{\prime}}{\rightarrow} G_{i, j-1},
$$

and the value $\sigma_{i j}$, determines $G_{i j} \in V$ with markings $M \in S\left(m\left(G_{i, j-1}, G_{i j}\right)\right)$ and $M^{\prime} \in S\left(m^{\prime}\left(G_{i-1, j}, G_{i j}\right)\right)$.

This is depicted below. Use $z, y, x, w$ to denote $G_{i-1, j-1}, G_{i-1, j}, G_{i, j-1}, G_{i j}$ for convenience and write $c=\sigma_{i j}$. In later examples we shall indicate $\sigma_{i j}=1$ by the symbol $\otimes$ and $\sigma_{i j}=0$ by a blank.
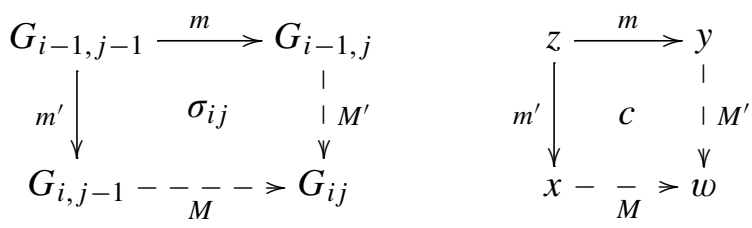

The local rule is defined using $\Phi$.

(1) If $z=x=y$ :

(a) If $c=0$, set $w=z$.

(b) If $c \neq 0$, let $\Phi_{x}(c)=\left(w, M, M^{\prime}\right)$. 
(2) If $z \neq x=y$ then let $\Phi_{x}\left(z, m, m^{\prime}\right)=\left(w, M, M^{\prime}\right)$.

(3) If $z=y$ and $z \neq x$ then let $w=x$ and $M^{\prime}=m^{\prime}$.

(4) If $z=x$ and $z \neq y$ then let $w=y$ and $M=m$.

(5) If $z, x, y$ are all distinct, then let $\left(w, M, M^{\prime}\right)=\Phi_{x y}\left(z, m, m^{\prime}\right)$.

This uniquely determines $G$ [Fomin 1995]. Its south edge $G_{n}$ is a $\Gamma$-tableau $P$ and its east edge $G_{\bullet n}$ is a $\Gamma^{\prime}$-tableau $Q$, both of a common shape $v=G_{n n} \in V$ with $h(v)=n$. This well-defines a map $\mathrm{I}_{\Phi}$ as in (8).

For the inverse of $\mathrm{I}_{\Phi}$, let $v \in V$ be such that $h(v)=n$, and let $(P, Q) \in \mathscr{T}(\Gamma, v) \times$ $\mathscr{T}\left(\Gamma^{\prime}, v\right)$. To recover $\sigma \in P_{n}(r)$, we initialize the south and east edges of $G$ to $P$ and $Q$ respectively. Then for each $i, j$ and two by two subgraph as above, we apply the inverse of the above local rule. Given labeled edges

$$
x \stackrel{M}{\rightarrow} w \quad \text { and } \quad y \stackrel{M^{\prime}}{\rightarrow} w,
$$

it determines $z \in V$ and marked edges

$$
z \stackrel{m}{\rightarrow} y \quad \text { and } \quad z \stackrel{m^{\prime}}{\rightarrow} x
$$

and a value $c \in\{0\} \sqcup S(r)$, such that $c \neq 0$ if and only if $z=x=y \neq w$ and $\Phi_{z}^{-1}\left(w, M, M^{\prime}\right)=c$. The inverse local rule is defined as follows.

(1) If $x=y$ :

(a) If $w=x$, let $z=x$.

(b) If $w \neq x$ :

(i) If $c:=\Phi_{x}^{-1}\left(w, M, M^{\prime}\right) \in S(r)$ : let $z=x$.

(ii) Otherwise $\Phi_{x}^{-1}\left(w, M, M^{\prime}\right)=\left(z, m, m^{\prime}\right)$.

(2) If $w=x \neq y$, let $z=y$ and $m^{\prime}=M^{\prime}$.

(3) If $w=y \neq x$, let $z=x$ and $m=M$.

(4) If $x, y, w$ are all distinct, let $\left(z, m, m^{\prime}\right)=\Phi_{x y}^{-1}\left(w, M, M^{\prime}\right)$.

In all cases but (1)(b)(i) let $c=0$. Using the inverse local rule the rest of $G$ is defined [Fomin 1995] and one obtains a well-defined element $\sigma \in P_{n}(r)$.

Theorem 2.10 [Fomin 1995]. Let $\left(\Gamma, \Gamma^{\prime}\right)$ be a dual graded graph with differential coefficient $r$. Then for any differential bijection $\Phi$ for $\left(\Gamma, \Gamma^{\prime}\right)$, the above construction defines a Schensted bijection $\mathrm{I}_{\Phi}$ of the form (8).

We call $\mathrm{I}_{\Phi}$ is the Schensted bijection induced by the differential bijection $\Phi$.

Example 2.11. The differential bijection $\Phi$ of Example 2.9 induces Schensted's row insertion bijection [1961]. 
Remark 2.12. For the Kac-Moody dual graded graphs $\left(\Gamma_{w}(K), \Gamma_{s}(\Lambda)\right)$ there is a natural choice for the off-diagonal part of the differential bijection. If $v \neq w$ with $v, w \in W$ then $\Phi_{v w}$ is essentially obtained from [Humphreys 1990, Lemma 5.11], just as in the proof of Theorem 2.3. For $i \in \operatorname{Des}(v)$, the marked down-then-up path

$$
v \stackrel{m^{\prime}}{\longrightarrow} s_{i} v \stackrel{m}{\longrightarrow} s_{i} v s_{\alpha}=w
$$

maps to the marked up-then-down path

$$
v \stackrel{m}{\longrightarrow} v s_{\alpha} \stackrel{m^{\prime}}{\longrightarrow} s_{i} v s_{\alpha}=w .
$$

Here $m$ and $m^{\prime}$ denote edge markings, which in either case are selected from sets of size $m_{\Lambda}\left(s_{i} v, s_{i} v s_{\alpha}\right)=\left\langle\alpha^{\vee}, \Lambda\right\rangle=m_{\Lambda}\left(v, v s_{\alpha}\right)$ and $n_{K}\left(\Lambda_{i}\right)$ respectively.

Currently we are not aware of a general rule for $\Phi_{v}$ which exhibits the coefficient of $v$ in $(D U-U D) v$ as $\langle K, \Lambda\rangle$. In Section 3 we shall give a special case where the bijection $\Phi_{v}$ has been constructed explicitly.

2E. Automorphisms and mixed insertion. This section is a natural synthesis of the ideas of [Fomin 1994] and [Haiman 1989] which does not seem to have been written down before. We believe this construction is particularly interesting for Kac-Moody dual graded graphs (see also Section 4).

Let $\left(\Gamma, \Gamma^{\prime}\right)$ be a pair of dual graded graphs with $\Gamma=(V, E, m, h)$ and $\Gamma=$ $\left(V, E^{\prime}, m^{\prime}, h\right)$. Say that a permutation $\tau: V \rightarrow V$ is an automorphism of $\left(\Gamma, \Gamma^{\prime}\right)$ if (1) $h \circ \tau=h$, (2) $(x, y) \in E$ if and only if $(\tau(x), \tau(y)) \in E$, and in this case, $m(x, y)=m(\tau(x), \tau(y))$, and (3) $(x, y) \in E^{\prime}$ if and only if $(\tau(x), \tau(y)) \in E^{\prime}$, and in this case, $m^{\prime}(x, y)=m^{\prime}(\tau(x), \tau(y))$.

Given a differential bijection $\Phi$ for $\left(\Gamma, \Gamma^{\prime}\right)$, we define its twist $\Phi^{\tau}$ by $\tau$ as follows. For every $x, y \in V$ there are natural bijections $\tau: \mathcal{U}_{x y} \rightarrow \mathcal{U}_{\tau(x) \tau(y)}$ given by $\left(z, m, m^{\prime}\right) \mapsto\left(\tau(z), m, m^{\prime}\right)$ and $\tau: \mathscr{D} u_{x y} \rightarrow \mathscr{D} u_{\tau(x) \tau(y)}$ given by $\left(w, M, M^{\prime}\right) \mapsto$ $\left(\tau(w), M, M^{\prime}\right)$. Let $\tau: S(r) \rightarrow S(r)$ be the identity permutation. Then define $\Phi_{x y}^{\tau}=\tau^{-1} \circ \Phi_{\tau(x) \tau(y)} \circ \tau$. It is easy to verify that $\Phi^{\tau}$ is also a differential bijection for $\left(\Gamma, \Gamma^{\prime}\right)$.

Example 2.13. Let $\Gamma=\Gamma^{\prime}=\mathbb{Y}$ and $\operatorname{tr}: \mathbb{Y} \rightarrow \mathbb{Y}$ the automorphism of $(\Gamma, \Gamma)$ that transposes partition diagrams. Let $\Phi$ be the differential bijection in Example 2.9. Then $I_{\Phi^{\text {tr }}}$ is Schensted's column insertion bijection [Schensted 1961].

For the sequel we assume that $\tau$ has finite order $\kappa$. A $\Gamma$-tableau whose edges have an auxiliary marking parameter $p \in S(\kappa)=\{0,1, \ldots, \kappa-1\}$ is called a $\tau$ mixed $\Gamma$-tableau. Let $\mathscr{T}_{\tau}(\Gamma)$ be the set of $\tau$-mixed $\Gamma$-tableaux. Suppose $\tau^{\prime}$ is an automorphism of $\left(\Gamma, \Gamma^{\prime}\right)$ of order $\kappa^{\prime}$. Let $\left(\Gamma, \Gamma^{\prime} ; \tau, \tau^{\prime}\right)$ denote the pair of dual graded graphs given by $\Gamma$ and $\Gamma^{\prime}$ except that $\Gamma$-edges (resp. $\Gamma^{\prime}$-edges) are labeled by $(m, p)$ with $p \in S(\kappa)$ (resp. $\left(m^{\prime}, p^{\prime}\right)$ with $\left.p^{\prime} \in S\left(\kappa^{\prime}\right)\right)$ and $m$ (resp. $\left.m^{\prime}\right)$ is a usual 
edge label for $\Gamma$ (resp. $\Gamma^{\prime}$ ). This multiplies the number of markings for each edge of $\Gamma$ (resp. $\Gamma^{\prime}$ ) by $\kappa$ (resp. $\left.\kappa^{\prime}\right)$. The differential coefficient of $\left(\Gamma, \Gamma^{\prime} ; \tau, \tau^{\prime}\right)$ is $r \kappa \kappa^{\prime}$ where $r$ is the differential coefficient of $\left(\Gamma, \Gamma^{\prime}\right)$.

Let $\Phi$ be a differential bijection for $\left(\Gamma, \Gamma^{\prime}\right)$. Then there is an obvious differential bijection (also denoted $\Phi$ ) for $\left(\Gamma, \Gamma^{\prime} ; \tau, \tau^{\prime}\right)$, defined by a trivial scaling by $\kappa$ in $\Gamma$ and by $\kappa^{\prime}$ in $\Gamma^{\prime}$.

We define another bijection

$$
P_{n}\left(\kappa \kappa^{\prime} r\right) \rightarrow \bigsqcup_{\substack{x \in V \\ h(x)=n}} \mathscr{T}_{\tau}(\Gamma, x) \times \mathscr{T}_{\tau^{\prime}}\left(\Gamma^{\prime}, x\right),
$$

called $\left(\tau, \tau^{\prime}\right)$-mixed insertion by modifying the process in which we construct the matrix $G_{i j}$ from the colored permutation $\sigma$. Instead of using the same differential bijection $\Phi$ to compute each $G_{i j}$, we use twists of $\Phi$ by automorphisms that depend on $(i, j)$.

Let $\sigma \in P_{n}\left(\kappa \kappa^{\prime} r\right)$. Regard $\sigma$ as a monomial matrix in which each nonzero entry has three labels $\left(c, p, p^{\prime}\right) \in S(r) \times S(\kappa) \times S\left(\kappa^{\prime}\right)$, where $S(\kappa)=\{0,1, \ldots, \kappa-1\}$ and $S\left(\kappa^{\prime}\right)=\left\{0,1, \ldots, \kappa^{\prime}-1\right\}$. Each horizontal (resp. vertical) edge is marked by a pair $(m, p)$ (resp. $\left.\left(m^{\prime}, p^{\prime}\right)\right)$ where $m$ (resp. $\left.m^{\prime}\right)$ is the usual marking and $p \in S(\kappa)$ (resp. $\left.p^{\prime} \in S\left(\kappa^{\prime}\right)\right)$. Let $(z, y, x, w)=\left(G_{i-1, j-1}, G_{i-1, j}, G_{i, j-1}, G_{i j}\right)$ and suppose that

$$
z \stackrel{(m, p)}{\longrightarrow} y \quad \text { and } \quad z \stackrel{\left(m^{\prime}, p^{\prime}\right)}{\longrightarrow} x
$$

are given, where it is understood that if $z=y(\operatorname{resp} z=x)$ then $(m, p)$ (resp. $\left.\left(m^{\prime}, p^{\prime}\right)\right)$ need not be specified. Then $G_{i j}$ is determined as before, except that instead of using $\Phi_{x y}$ we use the twist

$$
\Phi_{x y}^{\tau^{k}\left(\tau^{\prime}\right)^{k^{\prime}}}
$$

where $k$ and $k^{\prime}$ are as follows. Let $\left(c, p, p^{\prime}\right)$ be the nonzero entry of $\sigma$ in the $i$-th row, say, $\sigma_{i l}$. We set $k^{\prime}=p^{\prime}$. Separately, let $\left(c, p, p^{\prime}\right)$ be the nonzero entry of $\sigma$ in the $j$-th column, say, $\sigma_{q j}$. We set $k=p$. Note that in the case $q>i$ (resp. $l>j$ ) the bijection $\Phi_{x y}$ is not used in the local rule so the value of $k$ (resp. $k^{\prime}$ ) does not affect the algorithm.

In other words, if $\sigma_{i j}=\left(c, p, p^{\prime}\right)$ is a nonzero entry, then $\left(\tau^{\prime}\right)^{p^{\prime}}$ acts everywhere to the right in the $i$-th row and all vertical edges to the right (those of the form $G_{i-1, l} \rightarrow G_{i l}$ for $l \geq j$ ) are given the auxiliary marking $p^{\prime}$, and $\tau^{p}$ acts everywhere below in the $j$-th column, and all horizontal edges below (those of the form $G_{l, j-1} \rightarrow G_{l j}$ for $l \geq i$ ) are given the auxiliary marking $p$. The output is the pair $(P, Q) \in \mathscr{T}_{\tau}(\Gamma, v) \times \mathscr{T}_{\tau^{\prime}}\left(\Gamma^{\prime}, v\right)$ where $v=G_{n n}$ and $P$ and $Q$ are obtained from the south and east edges of $G$ respectively.

Proposition 2.14. $\left(\tau, \tau^{\prime}\right)$-mixed insertion gives a well-defined bijection (10). 
Example 2.15. In the context of Example 2.13, $\left(\tau, \tau^{\prime}\right)$-mixed insertion specializes to the following kinds of insertion algorithms, the first from [Schensted 1961] and the other three from [Haiman 1989].

(1) $\left(\tau, \tau^{\prime}\right)=(\mathrm{id}, \mathrm{id})$ : Schensted row insertion

(2) $\left(\tau, \tau^{\prime}\right)=($ id, tr): left-right insertion

(3) $\left(\tau, \tau^{\prime}\right)=(\operatorname{tr}, \mathrm{id}):$ mixed insertion

(4) $\left(\tau, \tau^{\prime}\right)=(\operatorname{tr}, \operatorname{tr})$ : doubly mixed insertion

2F. Restriction to parabolics. Let $J \subset I$. We say that a weight $\Lambda$ is supported on $J$ if $\Lambda=\sum_{j \in J} a_{j} \Lambda_{j}$. The Kac-Moody dual graded graphs $\left(\Gamma_{s}(\Lambda), \Gamma_{w}(K)\right)$ are compatible with restriction to parabolics. Let $W_{J} \subset W$ be the parabolic subgroup generated by $\left\{s_{j} \mid j \in J\right\}$ and let $W^{J}$ be the set of minimal length coset representatives in $W / W_{J}$. Note that $W^{J}$ inherits weak and strong orders from $W$ by restriction.

Proposition 2.16. Fix $J \subset I$. If $\Lambda$ is supported on $I \backslash J$ then the restriction of $\left(\Gamma_{s}(\Lambda), \Gamma_{w}(K)\right)$ to $W^{J}$ is a pair of dual graded graphs with differential coefficient $\langle K, \Lambda\rangle$.

Proof. Suppose $v \prec w$ is a weak cover. If $w \in W^{J}$ then $v \in W^{J}$ since $W^{J} \subset W$ is a lower order ideal for $\preceq$.

Suppose $w \lessdot v$ and $w \in W^{J}$ and $v \notin W^{J}$. Since $v$ has a reduced expression ending in $s_{j}$ for some $j \in J$ and $w$ is obtained from this reduced expression by omitting a simple generator, we conclude that $v=w s_{j}$. But $\Lambda$ is supported on $I \backslash J$, so $\left\langle\alpha_{j}^{\vee}, \Lambda\right\rangle=0$.

Combining these two facts we see that the proof of Theorem 2.3 restricts to $W^{J}$.

We shall use the following notation for maximal parabolic subgroups of $W$. For $i \in I$ we shall write $W^{i}$ for $W^{J}$ where $J=I \backslash\{i\}$. We denote by $\left(\Gamma_{s}(\Lambda), \Gamma_{w}(K)\right)^{i}=$ $\left(\Gamma_{s}^{i}(\Lambda), \Gamma_{w}^{i}(K)\right)$, the dual graded graph given by restricting $\left(\Gamma_{s}(\Lambda), \Gamma_{w}(K)\right)$ to $W^{i}$.

2G. The affine case. If the GCM $A$ is of finite type, then $Z^{+}=\{0\}$ and all of the edges of $\Gamma_{w}(K)$ are labeled 0 .

In this section let $A$ be of untwisted affine type. Let $0 \in I$ be the distinguished Kac 0 node and $J=I \backslash\{0\}$. Then $W$ is the affine Weyl group, $W_{J}=W_{\text {fin }}$ is the finite Weyl group, and we write $W^{0}=W^{J}$. By Proposition 2.16 the restriction of the Kac-Moody dual graded graph to $W^{0}$, is a dual graded graph. In this case the weak graph $\Gamma_{w}(K)$ has an interpretation involving the Schubert calculus of the homology of the affine Grassmannian, and the duality is a combinatorial expression of the pairing between the homology and cohomology of the affine Grassmannian. 
For affine algebras, $Z^{+}=\mathbb{Z}_{\geq 0} K$ where $K=K_{\text {can }}=\sum_{i \in I} k_{i} \alpha_{i}^{\vee}$ is the canonical central element; the vector $\left(k_{i}\right)_{i \in I}$ is the unique linear dependence of the rows of $A$ given by positive relatively prime integers [Kac 1990]. In this case, since the labels of $\Gamma_{w}(K)$ are linear in $K$, without loss of generality we shall only work with $K_{\text {can }}$ and define $\Gamma_{w}:=\Gamma_{w}\left(K_{\mathrm{can}}\right)$. The edge labels of $\Gamma_{w}$ are related to the homology multiplication in affine Grassmannians, as follows.

Let $\mathfrak{g}=\mathfrak{g}(A)$ be an untwisted affine algebra. Let $\mathrm{Gr}=\mathrm{Gr}_{\mathrm{G}}$ denote the affine Grassmannian of the simple Lie group $G$ whose Lie algebra $\mathfrak{g}_{\text {fin }}$ is the canonical simple Lie subalgebra of the affine algebra $\mathfrak{g}$. For $w \in W^{0}$ we let $\xi_{w} \in H_{*}(\mathrm{Gr})$ denote the corresponding homology Schubert class. Recall the constants $n(w, v)$ from (3).

Proposition 2.17. Let $\xi_{0}=\xi_{s_{0}}$ be the Schubert class indexed by the unique simple generator $s_{0} \notin W_{\text {fin. }}$. Then for every $w \in W^{0}$, we have in $H_{*}(\mathrm{Gr})$ the identity

$$
\xi_{0} \xi_{w}=\sum_{v} n(w, v) \xi_{v}
$$

where $v \in W^{0}$ runs over the weak covers $w \prec v$ of $w$.

Proof. We rely on the results of [Lam 2006] which in turn are based on unpublished work of Peterson. Let $S=\operatorname{Sym}\left(\left(\mathfrak{h}_{\mathbb{Z}}^{*}\right)_{\text {fin }}\right)=H^{T_{\text {fin }}}(\mathrm{pt})$ denote the symmetric algebra in the weights of the $\mathfrak{g}_{\mathrm{fin}}$ and $\phi_{0}: S \rightarrow \mathbb{Z}$ denote the evaluation at 0 . Let $\mathbb{A}_{0}$ denote the affine nilCoxeter algebra corresponding to $W$. As a free $\mathbb{Z}$-module $\mathbb{A}_{0}$ is spanned by elements $\left\{A_{w} \mid w \in W\right\}$ with multiplication given by

$$
A_{w} A_{v}= \begin{cases}A_{w v} & \text { if } \ell(w)+\ell(v)=\ell(w v) \\ 0 & \text { otherwise. }\end{cases}
$$

The affine nilHecke algebra $\mathbb{A}$ is the $\mathbb{Z}$-algebra generated by $\mathbb{A}_{0}$ and $S$ with the additional relation [Lam 2006, Lemma 3.1]

$$
A_{w} \lambda=(w \cdot \lambda) A_{w}+\sum_{w r_{\alpha} \lessdot w}\left\langle\lambda, \alpha^{\vee}\right\rangle A_{w} r_{\alpha},
$$

where $\alpha$ is always taken to be a positive root of $W$.

Now let

$$
\mathbb{B}=\left\{a \in \mathbb{A}_{0} \mid \phi_{0}(a s)=\phi_{0}(s) a \text { for any } s \in S\right\} \subset \mathbb{A}_{0}
$$

denote the affine Fomin-Stanley subalgebra, where $\phi_{0}: \mathbb{A} \rightarrow \mathbb{A}_{0}$ is given by $\phi_{0}\left(\sum_{w} a_{w} A_{w}\right)=\sum_{w} \phi_{0}\left(a_{w}\right) A_{w}$. Let $j_{0}: H_{*}\left(\mathrm{Gr}_{G}\right) \rightarrow \mathbb{B}$ denote the ring isomorphism [Lam 2006, Theorem 5.5] from the homology of $\mathrm{Gr}_{G}$ to the affine Fomin-Stanley algebra $\mathbb{B}$. We first show that $j_{0}\left(\xi_{0}\right)=\sum_{i \in I} k_{i} A_{i}$, where $A_{i}$ are the generators of the nilCoxeter algebra and $K_{\mathrm{can}}=\sum_{i} k_{i} \alpha_{i}^{\mathrm{V}}$. 
By [Lam 2006, Proposition 5.4], the element $j_{0}\left(\xi_{0}\right)$ is characterized by having unique Grassmannian term $A_{0}$, and the property that it lies in $\mathbb{B}$. Since $k_{0}=1$, the unique Grassmannian term property is immediate. Using (11), we calculate that

$$
\phi_{0}\left(a \alpha_{j}\right)=\sum_{i \in I} k_{i}\left\langle\alpha_{i}^{\vee}, \alpha_{j}\right\rangle=\left\langle K_{\mathrm{can}}, \alpha_{j}\right\rangle=0 .
$$

Since the $\alpha_{j}$ span $\left(\mathfrak{h}_{\mathbb{Z}}^{*}\right)_{\text {fin }}$ over $\mathbb{Q}$, we deduce that $\phi_{0}(a s)=\phi_{0}(s) a$ for any $s \in S$, and thus $j_{0}\left(\xi_{0}\right)=\sum_{i \in I} k_{i} A_{i}$. (This was first pointed out to us by Alex Postnikov.)

By [Lam 2006, Lemma 4.3, Theorem 5.5], we thus have

$$
\xi_{0} \xi_{w}=j\left(\xi_{0}\right) \cdot \xi_{w}=\left(\sum_{i \in I} k_{i} A_{i}\right) \cdot \xi_{w}=\sum_{w<s_{i} w} k_{i} \xi_{s_{i} w} .
$$

where we have used the action of $\mathbb{A}_{0}$ on $H_{*}\left(\mathrm{Gr}_{G}\right)$ given by

$$
A_{i} \cdot \xi_{w}= \begin{cases}\xi_{s_{i} w} & \text { if } s_{i} w>w \\ 0 & \text { otherwise }\end{cases}
$$

see [Lam 2006, (3.2)]. Recalling the definition $n\left(w, s_{i} w\right)=k_{i}$ of the weak graph $\Gamma_{w}$ from (3) this completes the proof.

\section{Affine type A and LLMS insertion}

For this section let $\mathfrak{g}(A)$ be the affine algebra of type $A_{n-1}^{(1)}$. In this case the combinatorics of the pair of dual graded graphs $\left(\Gamma_{s}\left(\Lambda_{i}\right), \Gamma_{w}\right)$ was studied extensively in [Lam et al. 2006] and was one of the main motivations of the current work. The affine insertion algorithm of [Lam et al. 2006] (which we shall call LLMS insertion) furnishes an explicit differential bijection for $\left(\Gamma_{s}\left(\Lambda_{i}\right), \Gamma_{w}\right)$. LLMS insertion involves nontrivial extensions of the notion of tableaux to semistandard weak and strong tableaux, and proves Pieri rules (formulae for certain Schubert structure constants) in the homology $H_{*}(\mathrm{Gr})$ and cohomology $H^{*}(\mathrm{Gr})$ of the affine Grassmannian of $\operatorname{SL}(n, \mathbb{C})$ [Lam et al. 2006].

For type $A_{n-1}^{(1)}$ the coefficients of the canonical central element $K$ are all 1 . Therefore the weak graph $\Gamma_{w}$ has all edge multiplicities equal to 1 . Using the rotational symmetry of the Dynkin diagram $A_{n-1}^{(1)}$, we may assume that $\Lambda=\Lambda_{0}$ and for brevity we write $\Gamma_{s}$ for $\Gamma_{s}\left(\Lambda_{0}\right)$.

Let $I=\{0,1, \ldots, n-1\}$ and let the Cartan matrix be defined by $a_{i, i+1}=a_{i+1, i}=$ -1 for all $i$, with indices taken modulo $n, a_{i i}=2$ for all $i \in I$, and $a_{i j}=0$ otherwise. As in Section 2B the Weyl group is defined by $m_{i, i+1}=3$ and $m_{i j}=2$ for $|i-j| \geq 2$.

3A. Affine permutations. We use the following explicit realization of the affine symmetric group $W=\widetilde{S}_{n}$. A bijection $w: \mathbb{Z} \rightarrow \mathbb{Z}$ is an affine permutation with period $n$ if $w(i+n)=w(i)$ for each $i \in \mathbb{Z}$ and $\sum_{i=1}^{n}(w(i)-i)=0$. The set of affine 
permutations with period $n$ form a group isomorphic to $\widetilde{S}_{n}$, with multiplication given by function composition. The reflections $t_{i j}$ in $\widetilde{S}_{n}$ are indexed by a pair of integers $(i, j)$ satisfying $i<j$ and $i \neq j \bmod n$. Suppose $v \lessdot v t_{i j}=w$ is a cover in $\widetilde{S}_{n}$. Then the edge $(v, w)$ in $\Gamma_{s}$ has multiplicity equal to $\#\{k \in \mathbb{Z} \mid$ $v(i) \leq k<v(j)$ and $k=0 \bmod n\}$ [Lam et al. 2006].

3B. Action of $\tilde{\boldsymbol{S}}_{\boldsymbol{n}}$ on partitions. Given a partition $\lambda$, one may associate a bi-infinite binary word $p(\lambda)=p=\cdots p_{-1} p_{0} p_{1} \cdots$ called its edge sequence. The edge sequence $p(\lambda)$ traces the border of the (French) diagram of $\lambda$, going from northwest to southeast, such that every letter 0 (resp. 1) represents a south (resp. east) step, and some cell in the $i$-th diagonal is touched by the steps $p_{i-1}$ and $p_{i}$. Here the cell $(i, j)$ lies in row $i$ (where row indices increase from south to north), column $j$ (where column indices increase from west to east), and diagonal $j-i$.

The affine symmetric group $\widetilde{S}_{n}$ acts on partitions, since elements of $\widetilde{S}_{n}$ are certain permutations $\mathbb{Z} \rightarrow \mathbb{Z}$ and partitions can be identified with their edge sequences, which are certain functions $\mathbb{Z} \rightarrow\{0,1\}$. Then for $i \in \mathbb{Z} / n \mathbb{Z}, s_{i} \lambda$ is obtained by removing from $\lambda$ every $\lambda$-removable cell of residue $i$, and adding to $\lambda$ every $\lambda$ addable cell of residue $i$. Here the residue of a box $(i, j)$ is the diagonal index $j-i$ taken modulo $n$.

3C. Cores and affine Grassmannian permutations. Using the language of cores, we shall describe the combinatorics of the dual graded graph $\left(\Gamma_{s}, \Gamma_{w}\right)^{0}$ afforded by Proposition 2.16.

An $n$-ribbon is a skew partition diagram $\lambda / \mu$ (the difference of the diagrams of the partitions $\lambda$ and $\mu$ ) consisting of $n$ rookwise connected cells, all with distinct residues. We say that this ribbon is $\lambda$-removable and $\mu$-addable. An $n$-core is a partition that admits no removable $n$-ribbon. Since the removal of an $n$-ribbon is the same thing as exchanging bits $p_{i}=0$ and $p_{i+n}=1$ in the edge sequence for some $i$, it follows that $\lambda$ is a core if and only if for every $i$, the sequence $p^{(i)}(\lambda):=$ $\cdots p_{i-2 n} p_{i-n} p_{i} p_{i+n} p_{i+2 n} \cdots$ consisting of the subsequence of bits indexed by $i$ $\bmod n$, has the form $\cdots 1111100000 \cdots$. We denote the set of $n$-cores by $\mathscr{C}_{n}$.

Proposition 3.1 [Lam et al. 2006; Misra and Miwa 1990]. The map $w \mapsto w \cdot \varnothing$ is a bijection $c: \widetilde{S}_{n}^{0} \rightarrow \mathscr{C}_{n}$. Moreover, for $v, w \in \widetilde{S}_{n}^{0}$, we have $v \leq w$ if and only if $c(v) \subseteq c(w)$, and if $v \lessdot w$ then $c(w) / c(v)$ is a disjoint union of translates of some ribbon $R$, and the number of components of $c(w) / c(v)$ is equal to the multiplicity $m(v, w)$ in $\Gamma_{s}$.

We say that $\mu \in \mathscr{C}_{n}$ covers $\lambda \in \mathscr{C}_{n}$ if $c^{-1}(\mu)>c^{-1}(\lambda)$. Thus a standard strong tableau in $\Gamma_{s}$ is a sequence $\lambda=\lambda^{0} \subset \lambda^{1} \subset \cdots \subset \lambda^{l}=\mu$ such that $\lambda^{i}$ covers $\lambda^{i-1}$ and one of the components of $\lambda^{i} / \lambda^{i-1}$ has been marked. 
It is easy to show that a core cannot have both an addable and a removable cell of the same residue. Thus, in the special case that $v \lessdot s_{i} v=w$ for some $i \in I, c(w)$ is obtained from $c(v)$ by adding all $c(v)$-addable cells of residue $i$, and the ribbon $R$ of Proposition 3.1 must be a single box. In this case we say that $c(v) \subset c(w)$ is a weak cover.

3D. LLMS insertion. In [Lam et al. 2006], for the affine symmetric group $\widetilde{S}_{n}$, semistandard analogues of weak and strong tableaux were defined (for all of $\widetilde{S}_{n}$, not just $\widetilde{S}_{n}^{0}$ ), and an RSK correspondence was given between a certain set of biwords or matrix words, and pairs of tableaux, one semistandard weak and the other semistandard strong. Let us consider the following restriction of this bijection. We first restrict to "standard" tableau pairs, that is, the case in which the tableaux are weak and strong tableaux as defined in Section 2C. Next we take the parabolic restriction from $\widetilde{S}_{n}$ to $\widetilde{S}_{n}^{0}$. Let us denote the restricted bijection by $I_{\text {LLMS }}$.

Let $\Phi$ be the differential bijection for $\left(\Gamma_{s}, \Gamma_{w}\right)^{0}$ such that $\mathrm{I}_{\Phi}=I_{\text {LLMS }}$. We describe it explicitly.

For $u, v \in \widetilde{S}_{n}^{0}$ with $u \neq v$, the off-diagonal part $\Phi_{u v}$ of $\Phi$ coincides with the natural definition given in Remark 2.12. The diagonal part $\Phi_{v}$ for $v \in \widetilde{S}_{n}^{0}$ is specified as follows. Let $\lambda=c(v)$ be the $n$-core corresponding to $v$. If $\lambda \subset \mu$ is a weak cover then $\mu / \lambda$ consists of all the $\lambda$-addable cells of $\lambda$ which have a fixed residue. As $\mu$ varies over all the weak covers of $\lambda$ we obtain all the $\lambda$-addable cells in this way. Thus there is a natural identification of the set $\mathscr{D} \boldsymbol{U}_{v}$ with the set of $\lambda$-addable cells. Similarly $\mathcal{U}_{v}$ may be identified with the set of $\lambda$-removable cells. This given, we may use the differential bijection denoted $\Phi_{\lambda}$ in Example 2.9 for Young's lattice. This defines a differential bijection $\Phi$ for $\left(\Gamma_{s}, \Gamma_{w}\right)^{0}$.

Example 3.2. Figure 1 shows the calculation of $I_{\text {LLMS }}$ of the permutation $\sigma=$ 412635 (written here in one-line notation; it corresponds to the permutation matrix with ones located positions $(i, \sigma(i))$ for $1 \leq i \leq 6)$ for $\widetilde{S}_{3}$. The symbols $\otimes$ encode $\sigma$ as described above Equation (9). Each arrow indicates a marked strong cover; the subscript 2 indicates that the marked component is the second from the southeast, and no subscript means the marked component is the southeastmost. Stars in the $P$ tableau indicate the marked components.

Remark 3.3. As $n$ goes to infinity, $\left(\Gamma_{s}, \Gamma_{w}\right)^{0}$ converges to the dual graded graph $(\mathbb{Y}, \mathbb{Y})$ of Example 2.2 and LLMS insertion converges to Schensted row insertion [Lam et al. 2006] because the respective differential bijections coincide in the limit.

\section{Folding}

An automorphism of the GCM $B=\left(b_{i j} \mid i, j \in J\right)$ is a permutation $\pi$ of $J$ such that $b_{\pi(i) \pi(j)}=b_{i j}$ for all $i, j \in J$. The automorphism $\pi$ is admissible if $b_{i j}=0$ for all $i$ and $j$ in the same $\pi$-orbit. 
$\otimes$

$\otimes$

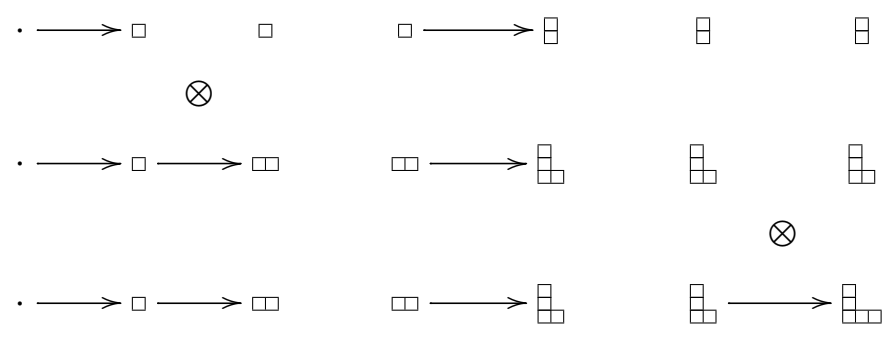

$\otimes$

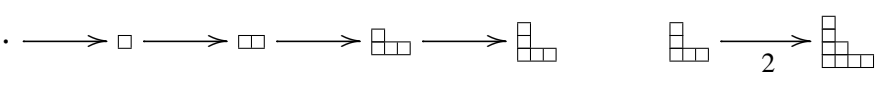

$\otimes$
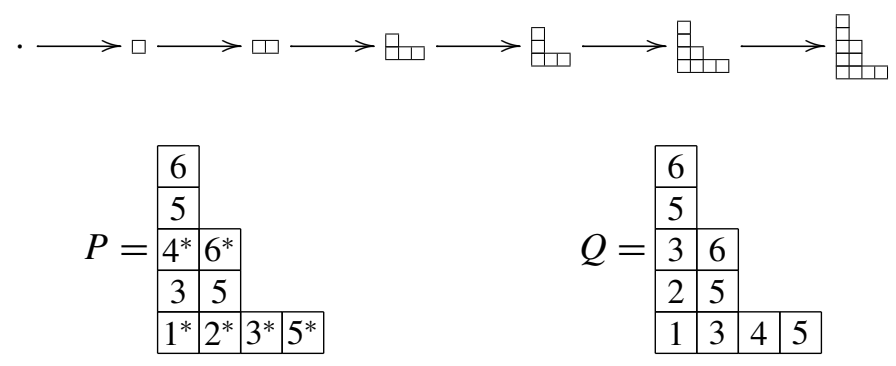

Figure 1. Growth diagram for LLMS insertion of 412635 for $\widetilde{S}_{3}$.

A GCM $A=\left(a_{i j} \mid i, j \in I\right)$ is symmetric if it is a symmetric matrix. It is symmetrizable if there are positive integers $o_{i}$ for $i \in I$, such that $D A$ is symmetric, where $D$ is the diagonal matrix with diagonal entries $o_{i}$.

Lusztig [1993] showed that every symmetrizable GCM $A$ can be constructed from a symmetric GCM $B$ that is equipped with an admissible automorphism $\pi$. We call this construction folding.

For $A$ and $B$ related in this manner, we show that the structure of every dual graded graph of the form $\left(\Gamma_{s}^{A}\left(\Lambda_{i^{\prime}}\right), \Gamma_{s}^{A}(K)\right)$ for $\mathfrak{g}(A)$, is encoded by some dual graded graph for $\mathfrak{g}(B)$. Thus the combinatorics for $\mathfrak{g}(A)$ is reduced to that of $\mathfrak{g}(B)$. In particular, for any affine algebra $\mathfrak{g}(A)$ there is a simply-laced affine algebra $\mathfrak{g}(B)$ related by folding, so that all affine Schensted bijections can be realized using only the simply-laced affine algebras. 
4A. Folding data. Let $B=\left(b_{i j} \mid i, j \in J\right)$ be a GCM and $\pi$ an admissible automorphism of $B$. Let $I$ be a set which indexes the $\pi$-orbits of $J$; we write $O_{i} \subset J$ for the $\pi$-orbit indexed by $i \in I$. Let $o_{i}=\left|O_{i}\right|$. It is easy to show that the matrix $A=\left(a_{i^{\prime} i} \mid i^{\prime}, i \in I\right)$ defined by

$$
a_{i^{\prime} i}=\frac{o_{i^{\prime}}}{o_{i}} \sum_{j \in O_{i}} b_{j^{\prime} j} \quad \text { for any } j^{\prime} \in O_{i^{\prime}},
$$

is a well-defined GCM. We say that $A$ is obtained from $(B, \pi)$ by folding.

Proposition 4.1 [Lusztig 1993, Proposition 14.1.2]. Given any symmetrizable GCM A, there is a symmetric GCM B with admissible automorphism $\pi$, such that $A$ is obtained from $(B, \pi)$ by folding. In particular, if $A$ is of affine type then $B$ can be taken to be of simply-laced affine type.

For the Kac-Moody algebras $\mathfrak{g}(A)$ and $\mathfrak{g}(B)$, we denote their weight lattices by $P_{A}$ and $P_{B}$, their coweight lattices by $P_{A}^{\vee}$ and $P_{B}^{\vee}$ and their coroot lattices by $Q_{A}^{\vee}$ and $Q_{B}^{\vee}$. For simplicity, we let $\Lambda_{i}, \alpha_{i}, \alpha_{i}^{\vee}$ be the fundamental weights, simple roots, and simple coroots for $\mathfrak{g}(A)$ and write $\omega_{i}, \beta_{i}, \beta_{i}^{\vee}$ for the corresponding data for $\mathfrak{g}(B)$.

Let $P_{A}^{\prime}=P_{A} /\left(\bigoplus_{i \in I} \mathbb{Z} \alpha_{i}^{\vee}\right)^{0}$ be the weight lattice of $A$ modulo the annihilator of the coroots $\left\{\alpha_{i}^{\vee}\right\}$. Similarly define $P_{B}^{\prime}$. Note that

$$
\alpha_{i}=\sum_{i^{\prime} \in I} a_{i^{\prime} i} \Lambda_{i^{\prime}} \quad \text { and } \quad \beta_{j}=\sum_{j^{\prime} \in J} b_{j^{\prime} j} \omega_{j^{\prime}},
$$

where $\alpha_{i}, \Lambda_{i}$ and $\beta_{j}, \omega_{j}$ also denote their respective images inside $P_{A}^{\prime}$ and $P_{B}^{\prime}$. Set $\kappa=\operatorname{lcm}_{i \in I}\left(o_{i}\right)$, and define $\psi: P_{A}^{\prime} \rightarrow P_{B}^{\prime}$ by

$$
\psi\left(\Lambda_{i}\right)=\frac{\kappa}{o_{i}} \widetilde{\omega}_{i},
$$

where $\widetilde{\omega}_{i}=\sum_{j \in O_{i}} \omega_{j}$ and $\widetilde{\beta}_{i}=\sum_{j \in O_{i}} \beta_{j}$ for $i \in I$. We have

$$
\begin{aligned}
\psi\left(\alpha_{i}\right) & =\sum_{i^{\prime}} a_{i^{\prime} i} \psi\left(\Lambda_{i^{\prime}}\right)=\sum_{i^{\prime}} a_{i^{\prime} i} \frac{\kappa}{o_{i^{\prime}}} \sum_{j^{\prime} \in O_{i^{\prime}}} \omega_{j^{\prime}} \\
& =\frac{\kappa}{o_{i}} \sum_{i^{\prime}} \sum_{j \in O_{i}} \sum_{j^{\prime} \in O_{i^{\prime}}} b_{j^{\prime} j} \omega_{j^{\prime}}=\frac{\kappa}{o_{i}} \widetilde{\beta}_{i},
\end{aligned}
$$

by (13) and (12). Define $\varphi: Q_{A}^{\vee} \rightarrow Q_{B}^{\vee}$ by

$$
\varphi\left(\alpha_{i}^{\vee}\right)=\widetilde{\beta}_{i}^{\vee}=\sum_{j \in O_{i}} \beta_{j} .
$$


4B. Weyl groups. For $i \in I$ define

$$
f\left(s_{i}^{A}\right)=\prod_{j \in O_{i}} s_{j}^{B} \in W_{B} .
$$

Since $\pi$ is admissible the reflections $\left\{s_{j}^{B} \mid j \in O_{i}\right\}$ commute with each other so that the product in (17) is independent of the order of its factors.

Steinberg [1968] showed that there is an embedding of Weyl groups $W_{A} \rightarrow W_{B}$. By [Nanba 2005] it respects the Bruhat order.

\section{Theorem 4.2.}

(1) [Steinberg 1968] There is an injective group homomorphism $f: W_{A} \rightarrow W_{B}$ defined by (17), whose image is the subgroup $W_{B}^{\pi}$ of $\pi$-fixed elements in $W_{B}$, where $\pi$ acts on $W_{B}$ by $\pi\left(s_{i}\right)=s_{\pi(i)}$.

(2) [Nanba 2005, Proposition 3.3 and Theorem 1.2] $v \leq w$ in $W_{A}$ if and only if $f(v) \leq f(w)$ in $W_{B}$. Moreover, if $w=s_{i_{1}} \cdots s_{i_{N}}$ is a reduced decomposition in $W_{A}$ then $f(w)=f\left(s_{i_{1}}\right) f\left(s_{i_{2}}\right) \cdots f\left(s_{i_{N}}\right)$ is a length-additive factorization in $W_{B}$.

\section{Corollary 4.3.}

(1) $\pi$ acts on $\Delta_{\mathrm{re}}^{+}(\mathfrak{g}(B))$.

(2) Suppose $v \lessdot w=v s_{\alpha}$ in $W_{A}$ for $\alpha \in \Delta_{\mathrm{re}}^{+}(\mathfrak{g}(A))$. Then there is a unique $\pi$ orbit $O \subset \Delta_{\mathrm{re}}^{+}(\mathfrak{g}(B))$ such that the reflections $\left\{s_{\gamma} \mid \gamma \in O\right\}$ commute, $f\left(s_{\alpha}\right)=$ $\prod_{\gamma \in O} s_{\gamma}$, and there is an isomorphism of the boolean lattice of subsets $O^{\prime}$ of $O$ with the interval $[f(v), f(w)]$ in $\left(W_{B}, \leq\right)$ given by $O^{\prime} \mapsto f(v) \prod_{\gamma \in O^{\prime}} s_{\gamma}$. We call $O$ the orbit associated with the cover $v \lessdot w$.

(3) Let $w \in W_{A}$ and $\gamma \in \Delta_{\text {re }}^{+}(\mathfrak{g}(B))$ be such that $f(w) s_{\gamma} \lessdot f(w)$ in $W_{B}$, and let $O \subset \Delta_{\mathrm{re}}^{+}(\mathfrak{g}(B))$ be the $\pi$-orbit of $\gamma$. Then there is a covering relation $v \lessdot w$ of $w$ in $W_{A}$ of which $O$ is the associated orbit.

(4) Let $v \in W_{A}, \gamma \in \Delta_{\mathrm{re}}^{+}(\mathfrak{g}(B))$ be such that $f(v) \lessdot f(v) s_{\gamma}$, and $O \subset \Delta_{\mathrm{re}}^{+}(\mathfrak{g}(B))$ the $\pi$-orbit of $\gamma$. Then there is a covering relation $v \lessdot w$ in $W_{A}$ of which $O$ is the associated cover.

Proof. For (1), let $\gamma \in \Delta_{\mathrm{re}}^{+}(\mathfrak{g}(B))$, with $\gamma=\tilde{u} \beta_{j}$ for some $\tilde{u} \in W_{B}$ and $j \in J$. Then $\pi(\gamma)=\pi(\tilde{u}) \beta_{\pi(j)} \in \Delta_{\mathrm{re}}(\mathfrak{g}(B))$ and $\pi$ clearly preserves the set of positive roots, so that $\pi$ acts on $\Delta_{\text {re }}^{+}(\mathfrak{g}(B))$.

For (2), there is a unique length-additive factorization $w=u_{1} s_{i} u_{2}$ in $W_{A}$ such that $v=u_{1} u_{2}$. We have $\alpha=u \alpha_{i}$ and $s_{\alpha}=u s_{i} u^{-1}$ where $u=u_{2}^{-1}$. Define $O=$ $\left\{f(u) \beta_{j} \mid j \in O_{i}\right\}$; since $f(u)$ is $\pi$-invariant, we have $\pi\left(f(u) \beta_{j}\right)=\pi(f(u)) \beta_{\pi(j)}=$ $f(u) \beta_{\pi(j)} \in O(\alpha)$, so that $O$ is a $\pi$-orbit. For $j \in O_{i}$ we have the relation $s_{f(u) \beta_{j}}=f(u) s_{j} f(u)^{-1}$, so the reflections $\left\{s_{\gamma} \mid \gamma \in O\right\}$ commute, being conjugate 
to commuting reflections $\left\{s_{j} \mid j \in O_{i}\right\}$. Since $f$ is a homomorphism we have $f\left(s_{\alpha}\right)=f(u)\left(\prod_{j \in O_{i}} s_{j}\right) f(u)^{-1}=\prod_{j \in O_{i}} f(u) s_{j} f(u)^{-1}=\prod_{\gamma \in O(\alpha)} s_{\gamma}$.

By Theorem $4.2 f(w)=f\left(u_{1}\right)\left(\prod_{j \in O_{i}} s_{j}\right) f\left(u_{2}\right)$ and $f(v)=f\left(u_{1}\right) f\left(u_{2}\right)$ are length-additive factorizations. It follows that there is an isomorphism of the boolean lattice of subsets $S \subset O_{i}$ with $[f(v), f(w)]$ where $S \mapsto f\left(u_{1}\right)\left(\prod_{j \in S} s_{j}\right) f\left(u_{2}\right)$. The desired isomorphism is given by sending $S \rightarrow O^{\prime}$ where $O^{\prime}=\left\{f(u) \beta_{j} \mid j \in S\right\}$.

For (3), let $w=s_{i_{1}} \cdots s_{i_{k}}$ be a reduced decomposition. Then the image $f(w)=$ $f\left(s_{i_{1}}\right) \cdots f\left(s_{i_{k}}\right)$ is length-additive by Theorem 4.2. Therefore the cover $f(w) s_{\gamma} \lessdot$ $f(w)$ is obtained by removing some unique reflection in $f\left(s_{i_{r}}\right)$ for some unique $r$. Let $u_{1}=s_{i_{1}} \cdots s_{i_{r-1}}, i=i_{r}$, and $u^{-1}=u_{2}=s_{i_{r+1}} \cdots s_{i_{k}}$. Letting $\alpha=u \alpha_{i} \in \Delta_{\mathrm{re}}^{+}(\mathfrak{g}(A))$ we find that the $\pi$-orbit $O$ of $\gamma$ is the orbit associated with the cover $v \lessdot w$ where $v=u_{1} u_{2}$.

The proof of (4) is similar.

The following result is proved similarly.

Corollary 4.4. Let $v \prec s_{i} v$ in $W_{A}$ for some $i \in I$. Then there is a poset isomorphism from the boolean lattice of subsets $O^{\prime}$ of $O_{i}$, to the interval $\left[f(v), f\left(s_{i} v\right)\right]$ of $\left(W_{B}, \preceq\right)$ given by $O^{\prime} \mapsto\left(\prod_{j \in O^{\prime}} s_{j}\right) f(v)$.

4C. Pairings. The action of $W_{B}$ on $P_{B}$ descends to $P_{B}^{\prime}$, and similarly for $W_{A}$.

\section{Theorem 4.5.}

(1) For all $\alpha^{\vee} \in Q_{A}^{\vee}$ and $\lambda \in P_{A}^{\prime}$ we have

$$
\left\langle\varphi\left(\alpha^{\vee}\right), \psi(\lambda)\right\rangle=\kappa\left\langle\alpha^{\vee}, \lambda\right\rangle .
$$

(2) For all $\Lambda \in P_{A}^{\prime}$ and $w \in W_{A}$ we have

$$
\psi(w \Lambda)=f(w) \psi(\Lambda) .
$$

(3) Let $w \in W_{A}, \Lambda \in P_{A}^{\prime}$ and $i \in I$. Then

$$
\left\langle\varphi\left(\alpha^{\vee}\right), f(w) \psi(\Lambda)\right\rangle=\kappa\left\langle\alpha^{\vee}, w \Lambda\right\rangle
$$

and in particular we have

$$
\left\langle\widetilde{\beta}_{i}^{\vee}, f(w) \psi(\Lambda)\right\rangle=\kappa\left\langle\alpha_{i}^{\vee}, w \Lambda\right\rangle .
$$

(4) The map $\varphi$ sends $Q_{A}^{\vee} \cap Z(\mathfrak{g}(A))$ into $Z(\mathfrak{g}(B))$.

Proof. By linearity it suffices to check (18) for $\alpha^{\vee}=\alpha_{i}^{\vee}$ and $\lambda=\Lambda_{k}$ for $i, k \in I$. We have

$$
\left\langle\varphi\left(\alpha_{i}^{\vee}\right), \psi\left(\Lambda_{k}\right)\right\rangle=\frac{\kappa}{o_{k}}\left\langle\widetilde{\beta}_{i}^{\vee}, \widetilde{\omega}_{k}\right\rangle=\frac{\kappa}{o_{k}} o_{i} \delta_{i k}=\kappa \delta_{i k}=\kappa\left\langle\alpha_{i}^{\vee}, \Lambda_{k}\right\rangle .
$$

This implies (18). 
It suffices to prove (19) for $w=s_{i^{\prime}}^{A}$ and $\Lambda=\Lambda_{i}$. For $i=i^{\prime}$ we have

$$
\psi\left(s_{i} \Lambda_{i}\right)=\psi\left(\Lambda_{i}-\alpha_{i}\right)=\frac{\kappa}{o_{i}}\left(\widetilde{\omega}_{i}-\widetilde{\beta}_{i}\right)
$$

by (14) and (15). Since $\pi$ is admissible, $\left(\prod_{j \in O_{i}} s_{j}^{B}\right) \widetilde{\omega}_{i}=\widetilde{\omega}_{i}-\widetilde{\beta}_{i}$, giving the required result. The case that $i \neq i^{\prime}$ is even easier.

Equation (20) follows immediately from (19) and (18) with $\lambda=w \Lambda$. Equation (21) is implied by (20) with $\alpha=\alpha_{i}$ and (16).

Let $K \in Q_{A}^{\vee}$. For any $i \in I$ and $j \in O_{i}$, by (18), (15), and the $\pi$-invariance of $\varphi(K)$, we have

$$
\kappa\left\langle\varphi(K), \beta_{j}\right\rangle=\frac{\kappa}{o_{i}}\left\langle\varphi(K), \widetilde{\beta}_{i}\right\rangle=\kappa\left\langle K, \alpha_{i}\right\rangle .
$$

It follows that $\varphi$ sends $Z(\mathfrak{g}(A))$ into $Z(\mathfrak{g}(B))$.

Remark 4.6. Let $A$ be a GCM of affine type, obtained as in [Lusztig 1993] by folding $(B, \pi)$. Then the canonical central elements and null roots are related by $\varphi\left(K^{A}\right)=K^{B}$ and $\psi\left(\delta^{A}\right)=r^{\vee} \delta^{B}$ where $r^{\vee}$ is the "twist" of the dual affine root system $X_{N}^{\left(r^{\vee}\right)}$ to that of $A$ in the nomenclature of [Kac 1990].

4D. Folding and insertion. Let $A=\left(a_{i j} \mid i, j \in I\right)$ be a GCM with associated KacMoody algebra $\mathfrak{g}(A), i^{\prime} \in I$, and $K \in Z^{+}(\mathfrak{g}(A))$. Suppose $A$ is obtained by folding the GCM $B=\left(b_{i j} \mid i, j \in J\right)$ with admissible automorphism $\pi$. Choose $j^{\prime} \in O_{i^{\prime}}$. We shall construct the dual graded graph $\left(\Gamma_{s}^{A}\left(\Lambda_{i^{\prime}}\right), \Gamma_{w}^{A}(K)\right)$ from the dual graded graph $\left(\Gamma_{s}^{B}\left(\omega_{j^{\prime}}\right), \Gamma_{w}^{B}(\varphi(K))\right)$. The construction only requires the subset $W_{B}^{\pi} \subset W_{B}$ of $\pi$-invariant vertices, and the edges incident to them, grouped according to their $\pi$-orbits.

Remark 4.7. The choice of $j^{\prime} \in O_{i^{\prime}}$ is immaterial; if one chooses another element of $O_{i^{\prime}}$ then the resulting type $B$ structures are transported to each other by a power of the automorphism $\pi$.

Proposition 4.8. Let $v \lessdot w$ in $W_{A}$ with $\alpha \in \Delta_{\mathrm{re}}^{+}(\mathfrak{g}(A))$ such that $w=v s_{\alpha}$ and let $O \subset \Delta_{\mathrm{re}}^{+}(\mathfrak{g}(B))$ be the associated orbit of the cover $v \lessdot w$, defined in Corollary 4.3. Then the following sets have the same cardinality.

(1) Marked edges $v \stackrel{m}{\rightarrow} w$ in $\Gamma_{s}^{A}\left(\Lambda_{i^{\prime}}\right)$.

(2) The disjoint union over $\gamma \in O$ of the sets $\left\{f(w) s_{\gamma} \stackrel{M}{\rightarrow} f(w)\right\}$ of marked edges going into $f(w)$ in the interval $[f(v), f(w)]$ in $\Gamma_{s}^{B}\left(\omega_{j^{\prime}}\right)$.

(3) The disjoint union over $\gamma \in O$ of the sets $\left\{f(v) \stackrel{M}{\rightarrow} f(v) s_{\gamma}\right\}$ of marked edges coming out of $f(v)$ in the interval $[f(v), f(w)]$ in $\Gamma_{s}^{B}\left(\omega_{j^{\prime}}\right)$. 
Proof. By Corollary 4.3 the last two sets are in bijection. Let $u \in W_{A}$ and $i \in I$ be such that $\alpha=u \alpha_{i}$. Then $O=\left\{f(u) \beta_{j} \mid j \in O_{i}\right\}$. Using Theorem 4.5 and the $\pi$-invariance of $\sum_{\gamma \in O} \gamma^{\vee}$, we have

$$
\begin{aligned}
\sum_{\gamma \in O} m_{\omega_{j^{\prime}}}^{B}\left(f(w) s_{\gamma}, f(w)\right) & =\sum_{\gamma \in O}\left\langle\gamma^{\vee}, \omega_{j^{\prime}}\right\rangle=\frac{1}{o_{i^{\prime}}} \sum_{\gamma \in O}\left\langle\gamma^{\vee}, \widetilde{\omega}_{i^{\prime}}\right\rangle \\
& =\frac{1}{\kappa} \sum_{\gamma \in O}\left\langle\gamma^{\vee}, \psi\left(\Lambda_{i^{\prime}}\right)\right\rangle=\frac{1}{\kappa} \sum_{j \in O_{i}}\left\langle f(u) \beta_{j}^{\vee}, \psi\left(\Lambda_{i^{\prime}}\right)\right\rangle \\
& =\frac{1}{\kappa}\left\langle\widetilde{\beta}_{i}^{\vee}, f(u)^{-1} \psi\left(\Lambda_{i^{\prime}}\right)\right\rangle \\
& =\left\langle\alpha_{i}^{\vee}, u^{-1} \Lambda_{i^{\prime}}\right\rangle=\left\langle\alpha^{\vee}, \Lambda_{i^{\prime}}\right\rangle=m_{\Lambda_{i^{\prime}}}^{A}\left(w s_{\alpha}, w\right) .
\end{aligned}
$$

By definition the graded graph $\Gamma_{s}^{B}\left(\omega_{j^{\prime}}\right)^{\pi}$ has vertex set $f\left(W_{A}\right)=W_{B}^{\pi} \subset W_{B}$, grading function $h(f(v))=\ell(v)$ for $v \in W_{A}$, and for every cover $v \lessdot w$ in $W_{A}$, an edge from $f(v)$ to $f(w)$ whose multiplicity is the common number in Proposition 4.8. It is completely specified by the $\pi$-invariant elements of $\Gamma_{s}^{B}\left(\omega_{j^{\prime}}\right)$ and their incident edges.

Corollary 4.9. The graded graphs $\Gamma_{s}^{A}\left(\Lambda_{i^{\prime}}\right)$ and $\Gamma_{s}^{B}\left(\omega_{j^{\prime}}\right)^{\pi}$ are isomorphic.

Proof. By Theorem 4.2 the map $f: W_{A} \rightarrow W_{B}^{\pi}$ is a grade- and edge-preserving bijection. The edge multiplicities agree by Proposition 4.8.

We call $\Gamma_{s}^{B}\left(\omega_{j^{\prime}}\right)^{\tau}$ the folded strong graph and its tableaux folded strong tableaux.

Proposition 4.10. Let $v \in W_{A}$ and $i \in I \backslash \operatorname{Des}(v)$ so that $v \prec w=s_{i} v$. Fix any $j \in O_{i}$. Then the following sets have the same cardinality.

(1) The marked edges $v \stackrel{m^{\prime}}{\rightarrow} w$ in $\Gamma_{w}^{A}(K)$.

(2) The marked edges $f(v) \stackrel{M^{\prime}}{\rightarrow} s_{j} f(v)$ in $\Gamma_{w}^{B}(\varphi(K))$.

(3) The marked edges $s_{j} f(w) \stackrel{M^{\prime}}{\rightarrow} f(w)$ in $\Gamma_{w}^{B}(\varphi(K))$.

Proof. By a proof similar to the one for (22) and recalling the definition (3) we have

$$
n_{K}^{A}\left(v, s_{i} v\right)=\left\langle K, \Lambda_{i}\right\rangle=\left\langle\varphi(K), \omega_{j}\right\rangle=n_{\varphi(K)}^{B}\left(f(v), s_{j} f(v)\right) .
$$

This proves the proposition.

By definition the graded graph $\Gamma_{w}^{B}(\varphi(K))^{\pi}$ has vertex set $f\left(W_{A}\right)=W_{B}^{\pi}$, grading function $h(f(w))=\ell(w)$, and for each $v \in W_{A}$ and $i \in I \backslash \operatorname{Des}(v)$, an edge from $f(v)$ to $f\left(s_{i} v\right)$ whose multiplicity is the common multiplicity in Proposition 4.10.

Corollary 4.11. The graded graphs $\Gamma_{w}^{A}(K)$ and $\Gamma_{w}^{B}(\varphi(K))^{\pi}$ are isomorphic. 
We call $\Gamma_{w}^{B}(\varphi(K))^{\pi}$ the folded weak graph. Its tableaux are called folded weak tableaux.

Corollary 4.12. $\left(\Gamma_{s}^{B}\left(\omega_{j^{\prime}}\right)^{\pi}, \Gamma_{w}^{B}(\varphi(K))^{\pi}\right)$ and $\left(\Gamma_{s}^{A}\left(\Lambda_{i^{\prime}}\right), \Gamma_{w}^{A}(K)\right)$ are isomorphic dual graded graphs.

Proof. This follows immediately from Corollaries 4.9 and 4.11 .

Using a differential bijection $\Phi^{B}$ for $\left(\Gamma_{s}^{B}\left(\omega_{j^{\prime}}\right), \Gamma_{w}^{B}(\varphi(K))\right)$, we construct a differential bijection for the folded dual graded graph $\left(\Gamma_{s}^{B}\left(\omega_{j^{\prime}}\right)^{\pi}, \Gamma_{w}^{B}(\varphi(K))^{\pi}\right)$, which, by the identifications given in Propositions 4.8 and 4.10 , yields a differential bijection $\Phi^{A}$ for $\left(\Gamma_{s}^{A}\left(\Lambda_{i^{\prime}}\right), \Gamma_{w}^{A}(K)\right)$.

By Remark 2.12 the off-diagonal part $\Phi_{v w}^{A}$ for $v \neq w$ in $W_{A}$, has already been specified.

For the diagonal terms, since $\operatorname{Des}(f(v))=\bigsqcup_{i \in \operatorname{Des}(v)} O_{i}$, by (23) in the special case of a cover of the form $s_{i} v=v s_{\alpha}>v$ with $\alpha=v^{-1} \alpha_{i} \in \Delta_{\text {re }}^{+}$and (24) we have

$$
\begin{aligned}
\sum_{i \in I \backslash \operatorname{Des}(v)} m_{\Lambda_{i^{\prime}}}^{A}\left(v, s_{i} v\right) & n_{K}^{A}\left(v, s_{i} v\right) \\
& =\sum_{j \in J \backslash \operatorname{Des}(f(v))} m_{\omega_{j^{\prime}}}^{B}\left(f(v), s_{j} f(v)\right) n_{\varphi(K)}^{B}\left(f(v), s_{j} f(v)\right) .
\end{aligned}
$$

For $i \in \operatorname{Des}(v)$ and $j \in O_{i}$, we have $v s_{\alpha}=s_{i} v \prec v$ where $\alpha=v^{-1} \alpha_{i} \in-\Delta_{\text {re }}^{+}$, and an analogous computation yields

$$
\begin{aligned}
\sum_{i \in \operatorname{Des}(v)} m_{\Lambda_{i^{\prime}}}^{A}\left(s_{i} v, v\right) & n_{K}^{A}\left(s_{i} v, v\right) \\
& =\sum_{j \in \operatorname{Des}(f(v))} m_{\omega_{j^{\prime}}}^{B}\left(s_{j} f(v), f(v)\right) n_{\varphi(K)}^{B}\left(s_{j} f(v), f(v)\right) .
\end{aligned}
$$

Using the bijections of Propositions 4.8 and 4.10, we obtain bijections $\mathscr{D} U_{v}^{A} \rightarrow$ $\mathscr{D} u_{f(v)}^{B}$ and $u_{D_{v}^{A}}^{A} \rightarrow \mathcal{U D}_{f(v)}^{B}$. Under these identifications we obtain a differential bijection $\Phi^{A}$ for $\left(\Gamma_{s}^{A}\left(\Lambda_{i^{\prime}}\right), \Gamma_{w}^{A}(K)\right)$.

Proposition 4.13. Let the GCMA $=\left(a_{i j} \mid i, j \in I\right)$ be obtained by folding from the $G C M B=\left(b_{i j} \mid i, j \in J\right)$ with admissible automorphism $\pi$. Then for any $i^{\prime} \in I$ and $j^{\prime} \in O_{i^{\prime}}$, a differential bijection $\Phi^{B}$ for $\left(\Gamma_{s}^{B}\left(\omega_{j^{\prime}}\right), \Gamma_{w}^{B}(\varphi(K))\right.$ restricts to a differential bijection $\Phi^{A}$ for the pair of dual graded graphs $\left(\Gamma_{s}^{A}\left(\Lambda_{i^{\prime}}\right), \Gamma_{w}^{A}(K)\right)$.

We shall give an extensive example in Section 5.

Remark 4.14. It is possible to axiomatize conditions for an arbitrary pair of dual graded graphs $\left(\Gamma, \Gamma^{\prime}\right)$ and an automorphism $\pi$ of $\left(\Gamma, \Gamma^{\prime}\right)$ to give rise to a folded insertion in the manner we have described for Kac-Moody graded graphs. The key properties needed are abstract graph-theoretic formulations of Theorem 4.2 and Corollary 4.3. Since we have no interesting examples that do not come from Kac-Moody dual graded graphs, we will not make this precise. 


\section{Affine type $C$ combinatorics}

In this section we consider folded insertion for the affine root system $C_{n}^{(1)}$. Folding works for the entire Weyl group. However here we shall restrict our discussion to the explicit description of folded insertion for the maximal parabolic quotient of the affine Weyl group given by the dual graded graphs $\left(\Gamma_{s}\left(\Lambda_{i^{\prime}}\right), \Gamma_{w}(K)\right)^{i^{\prime}}$ using $2 n$-cores, where $i^{\prime} \in I$ is any Dynkin node. In the limit $n \rightarrow \infty$ one obtains a new Schensted bijection for every integer $i$, which for $i=0$ coincides with "standard" Sagan-Worley insertion into shifted tableaux.

Let $I=\{0,1, \ldots, n\}$ be the Dynkin node set and $\left(a_{i j}\right)$ the GCM, with $a_{i i}=2$ for $i \in I, a_{i, i+1}=a_{i+1, i}=-1$ for $1 \leq i \leq n-2, a_{01}=a_{n, n-1}=-1, a_{10}=a_{n-1, n}=-2$, and other entries zero. Using the recipe in Section 2B, the Weyl group $W_{n}$ has generators $s_{i}$ for $i \in I$ satisfying $s_{i}^{2}=1$ for $i \in I$ and $\left(s_{i} s_{j}\right)^{m_{i j}}=1$ for $i, j \in I$ with $i \neq j$, where $m_{01}=m_{n-1, n}=4, m_{i, i+1}=3$ for $1 \leq i \leq n-2$, and $m_{i j}=0$ for $|i-j| \geq 2$.

5A. Folding for $\boldsymbol{C}_{\boldsymbol{n}}^{(\mathbf{1})}$. Let $A=C_{n}^{(1)}$ and $B=A_{2 n-1}^{(1)}$ denote the two GCMs. We use the notation of Section 4.

Let $\pi$ be the admissible automorphism of $B$ given by $j \mapsto 2 n-j$ where indices are taken modulo $2 n$. We index the $\pi$-orbits by $O_{0}=\{0\}, O_{n}=\{n\}$, and $O_{i}=$ $\{i, 2 n-i\}$ for $i \in I \backslash\{0, n\}$. It is easy to check that $A$ is obtained from $(B, \pi)$ by folding.

Let $K$ be the canonical central element for $C_{n}^{(1)}$. Let $i^{\prime} \in I$ and $j^{\prime} \in O_{i^{\prime}}$. We define folded insertion for the dual graded graph $\left(\Gamma_{s}^{A}\left(\Lambda_{i^{\prime}}\right), \Gamma_{w}^{A}(K)\right)^{i^{\prime}}$, realized by LLMS insertion for $\left(\Gamma_{s}^{B}\left(\omega_{j^{\prime}}\right), \Gamma_{w}^{B}(\varphi(K))\right)$. We call this induced folded insertion the "LLMS insertion for $W_{n}^{i^{\prime}}$ " (even though it also depends on $j^{\prime}$ ).

5B. 2n-cores. As before, fix $i^{\prime} \in I$ and $j^{\prime} \in O_{i^{\prime}}$. The elements of the parabolic quotient $W_{n}^{i^{\prime}}$ may be realized by $2 n$-cores as follows.

By Proposition 3.1 there is a bijection $c: \widetilde{S}_{2 n}^{0} \rightarrow \mathscr{C}_{2 n}$. Using a rotational automorphism of the Dynkin diagram of type $A_{2 n-1}^{(1)}$, for any $k \in J$ one may define the $k$-action of $\widetilde{S}_{2 n}$ on $\mathscr{C}_{2 n}$, denoted $w \cdot{ }_{k} \lambda$, which is the same as before except that the diagonal of the cell $(i, j)$ is $j-i+k$. Since the stabilizer of $\varnothing$ under the $k$-action of $\widetilde{S}_{2 n}$ on $\mathscr{C}_{2 n}$ is $\left(\widetilde{S}_{2 n}\right)_{J \backslash\{k\}}$, there is a bijection $c_{k}: \widetilde{S}_{2 n}^{k} \rightarrow \widetilde{S}_{2 n} /\left(\widetilde{S}_{2 n}\right)_{J \backslash\{k\}} \rightarrow \mathscr{C}_{2 n}$ defined by $c_{k}(w)=w \cdot k \varnothing$.

Define the map $s c_{i^{\prime}}: W_{n} \rightarrow \mathscr{C}_{2 n}$ by $w \mapsto f(w) \cdot{ }_{j^{\prime}} \varnothing$, where $f: W_{n} \rightarrow \widetilde{S}_{2 n}$ is the Weyl group homomorphism of Section 4. Note that $f\left(W_{n}^{i^{\prime}}\right) \subset \widetilde{S}_{2 n}^{J \backslash O_{i^{\prime}}}$.

Denote by $\mathscr{C}_{2 n}^{j^{\prime}}$ the image of $s c_{i^{\prime}}$.

The following result is the $C_{n}^{(1)}$-analogue of (part of) Proposition 3.1.

Proposition 5.1. The map $s c_{i^{\prime}}$ restricts to a bijection $W_{n}^{i^{\prime}} \rightarrow \mathscr{C}_{2 n}^{j^{\prime}}$. For $v, w \in W_{n}^{i^{\prime}}$ we have $v \leq w$ if and only if $s c_{i^{\prime}}(v) \subset s c_{i^{\prime}}(w)$. 
Proof. The stabilizer of $W=W_{n}$ acting on $\varnothing$ is equal to $W_{I-\left\{i^{\prime}\right\}}$, so the first statement is immediate. Let $v, w \in W_{n}^{i^{\prime}}$. The following are equivalent: (1) $v \leq$ $w$; (2) $f(v) \leq f(w)$; (3) $f(v)\left(\widetilde{S}_{2 n}\right)_{J \backslash\{j\}} \leq f(w)\left(\widetilde{S}_{2 n}\right)_{J \backslash\{j\}}$ for all $j \in O_{i^{\prime}}$; (4) $f(v)\left(\widetilde{S}_{2 n}\right)_{J \backslash\left\{j^{\prime}\right\}} \leq f(w)\left(\widetilde{S}_{2 n}\right)_{J \backslash\left\{j^{\prime}\right\}} ;(5) s c_{i^{\prime}}(v) \subset s c_{i^{\prime}}(w)$. (1) and (2) are equivalent by Theorem 4.2. (2) and (3) are equivalent by Proposition 5.2 below applied to the data $f(v), f(w)$, and $J \backslash O_{i^{\prime}}$ in $\widetilde{S}_{2 n}$. Since $f(v)$ and $f(w)$ are $\pi$-invariant, (3) and (4) are equivalent, because $j^{\prime} \in O_{i^{\prime}}$ and the condition for $j$ is invariant as $j$ runs over a $\pi$-orbit. (4) and (5) are equivalent by Proposition 3.1.

For a Coxeter group $W$ and a parabolic subgroup $W_{J}$, the strong (Bruhat) order denoted $\leq$ on the quotient $W / W_{J}$ is the partial order naturally induced from the strong order on $W^{J}$. The following result is due to [Deodhar 1977].

Proposition 5.2. Let $W$ be a Coxeter group with simple generators indexed by $P$ and let $Q \subset P$. Suppose $x, y \in W^{Q}$. Then $x \leq y$ if and only if $x W_{Q^{\prime}} \leq y W_{Q^{\prime}}$ for every maximal parabolic subgroup $W_{Q^{\prime}} \supset W_{Q}$.

We describe $\mathscr{C}_{2 n}^{0}$ explicitly in Section 5D, together with an explicit description of LLMS insertion for $W_{n}^{0}$. It would be interesting to obtain an explicit description of $\mathscr{C}_{2 n}^{j^{\prime}}$ for arbitrary $j^{\prime}$. The explicit description of the Chevalley coefficients in a manner similar to Proposition 3.1, and of LLMS insertion for $W_{n}^{i^{\prime}}$ appears to be rather subtle.

5C. Large rank limit of folded LLMS insertion. We now consider the limit of LLMS insertion for $W_{n}^{i^{\prime}}$ as $n$ goes to $\infty$, in such a way that the nodes near 0 in $A_{2 n-1}^{(1)}$ are stable; for this purpose we label these nodes . . , $-2,-1,0,1,2, \ldots$.

Let $A_{ \pm \infty}$ be the Kac-Moody algebra ${ }^{1}$ whose Dynkin diagram has vertex set $J_{\infty}=\mathbb{Z}$, with Cartan matrix $\left(b_{i j}\right)$ such that $b_{i i}=2$ and $b_{i, i+1}=b_{i+1, i}=-1$, and $b_{i j}=0$ otherwise. Let $S_{ \pm \infty}$ be its Weyl group: it has generators $s_{j}$ for $j \in J_{\infty}$, with relations $s_{j}^{2}=1,\left(s_{j} s_{j+1}\right)^{3}=1$, and $\left(s_{i} s_{j}\right)^{2}=1$ for $|i-j| \geq 2$. Then $S_{ \pm \infty}$ acts on partitions: $s_{j} \cdot \lambda$ is obtained from $\lambda$ by adding the unique $\lambda$-addable cell in diagonal $j$ if it exists, and removing the unique $\lambda$-removable cell in diagonal $j$ if it exists (remembering the shift in diagonal index by $j^{\prime}$ ). Then $S_{ \pm \infty} \varnothing=\mathbb{Y}$ is the set of all partitions and there is a bijection $c_{j^{\prime}}: S_{ \pm \infty}^{j^{\prime}} \cong \mathbb{Y}$.

Let $C_{\infty}$ be the Kac-Moody algebra with Dynkin node set $I_{\infty}=\mathbb{Z}_{\geq 0}$ and Cartan matrix $a_{i j}$ with $a_{i i}=2$ for $i \in I_{\infty}, a_{i, i+1}=a_{i+1, i}=-1$ for $i \in I_{\infty} \backslash\{0\}, a_{01}=-1$ and $a_{10}=-2$. Then its Weyl group $W_{\infty}$ has generators $s_{i}$ for $i \in I_{\infty}$ with relations $s_{i}^{2}=1,\left(s_{0} s_{1}\right)^{4}=1,\left(s_{i} s_{i+1}\right)^{3}=1$ for $i \in I_{\infty} \backslash\{0\}$, and $\left(s_{i} s_{j}\right)^{2}=1$ for $|i-j| \geq 2$. As before, there is an injective homomorphism $f: W_{\infty} \rightarrow S_{ \pm \infty}$ given by $f\left(s_{0}\right)=s_{0}$ and $f\left(s_{i}\right)=s_{i} s_{-i}$ for $i>0$.

\footnotetext{
${ }^{1}$ We allow infinite Dynkin diagrams in a formal manner.
} 
Then $W_{\infty}$ acts on partitions via $f$. Define $\bigvee^{i^{\prime}}=W_{\infty} \cdot \varnothing$. The limit of Proposition 5.1 gives a bijection $s c_{i^{\prime}}: W_{\infty}^{i^{\prime}} \cong \mathbb{Y}^{i^{\prime}}$. The strong and weak orders on $\mathscr{C}_{2 n} \cong \widetilde{S}_{2 n}^{j^{\prime}}$ both converge to Young's lattice $\mathbb{Y}$. The weak order on $\mathscr{C}_{2 n}^{j^{\prime}} \cong W_{n}^{i^{\prime}}$ converges to the weak order on $\mathbb{Y}^{i^{\prime}} \cong W_{\infty}^{i^{\prime}}$, in which a cover $\lambda \subset s_{i} \lambda$ adds cells in diagonals $i$ and $-i$ if $i>0$ or just the cell in diagonal 0 if $i=0$.

Proposition 5.3. Suppose $\lambda \subset \mu$ is a strong cover in $\mathbb{Y}^{i^{\prime}} \cong W_{\infty}^{i^{\prime}}$ with $s c_{i^{\prime}}^{-1}(\lambda)=$ $w \lessdot w s_{\alpha}=s c_{i^{\prime}}^{-1}(\mu)$. If $s_{\alpha}$ is conjugate to $s_{0}$ then the Chevalley coefficient $\left\langle\alpha^{\vee}, \Lambda_{i^{\prime}}\right\rangle$ is equal to 1 and $\mu / \lambda$ has a single connected component which is necessarily a ribbon. Otherwise suppose $f\left(s_{\alpha}\right)=s_{\beta} s_{\beta^{\prime}}$. Then the Chevalley coefficient $\left\langle\alpha^{\vee}, \Lambda_{i^{\prime}}\right\rangle$ is equal 1 or 2 depending on whether one or both of $s c\left(w s_{\beta}\right)$ and $s c\left(w s_{\beta^{\prime}}\right)$ strictly contain $\lambda$. Furthermore, each strict containment has a single connected component which is a ribbon.

Proof. That the skew partitions in question contain a single connected component which equals a ribbon follows from the fact that they are obtained by the action of a reflection on a partition, which always changes the shape by a ribbon. This follows from the edge sequence discussion in Section 3.

Suppose $s_{\alpha}$ is conjugate to $s_{0}$ and $f\left(s_{\alpha}\right)=s_{\beta}$. Every strong cover in $\mathbb{Y}$ has Chevalley coefficient equal to 1 or 0 (since a single box is added). Now write $f(w)=x y$ so that $f\left(w s_{\alpha}\right)=x s_{0} y$ in $S_{ \pm \infty}$. The Chevalley coefficient $\left\langle\alpha^{\vee}, \Lambda_{i^{\prime}}\right\rangle$ is equal to the Chevalley coefficient $\left\langle\beta^{\vee}, \omega_{j^{\prime}}\right\rangle$ which is equal to the Chevalley coefficient of the cover $y \lessdot s_{0} y$ in $S_{ \pm \infty}$ (with respect to $\omega_{j^{\prime}}$ ). Since $\mu \neq \lambda$, we must have $y \cdot \varnothing \subsetneq\left(s_{0} y\right) \cdot \varnothing$. Thus the required Chevalley coefficient must be nonzero, and hence equal to 1 . We have used the calculation $\left\langle y^{-1} \beta_{0}^{\vee}, \omega_{j^{\prime}}\right\rangle=\left\langle\beta_{0}^{\vee}, y \omega_{j^{\prime}}\right\rangle=$ $\left\langle\beta_{0}^{\vee}, y^{\prime} \omega_{j^{\prime}}\right\rangle$, where $y^{\prime}=c_{j^{\prime}}^{-1}(y \cdot \varnothing)$ is the parabolic component" of $y$.

The proof for $f\left(s_{\alpha}\right)=s_{\beta} s_{\beta^{\prime}}$ follows in a similar manner. The only delicate issue is to show that if $\left\langle\beta^{\vee}, \omega_{j^{\prime}}\right\rangle=1$ then $\lambda \subset \operatorname{sc}\left(w s_{\beta}\right)$ is a strict inclusion. But $\left\langle\beta^{\vee}, \omega_{j^{\prime}}\right\rangle=1$ implies that $\left(s_{\beta} \cdot \varnothing\right) \neq \varnothing$ so that $\left(w s_{\beta} \cdot \varnothing\right) \neq(w \cdot \varnothing)$.

Figure 3 shows the case of a domino appearing in the strong tableau $P$, corresponding to the strong cover $s_{2} s_{0} s_{1} \lessdot s_{2} s_{1} s_{0} s_{1}$ in $W_{\infty}^{1}$.

In the case the Chevalley coefficient described in Proposition 5.3 is equal to 2, the difference $\mu / \lambda$ is a union of two ribbons, since $s_{\beta}$ and $s_{\beta^{\prime}}$ commute. We have not shown that these ribbons do not touch, so the difference $\mu / \lambda$ can potentially be written as the union of the two ribbons in two ways, corresponding to the left action of $s_{f(w) \beta} s_{f(w) \beta^{\prime}}$ and $s_{f(w) \beta^{\prime}} s_{f(w) \beta}$. To obtain a strong tableau in $\mathbb{Y}^{i^{\prime}}$, we must mark a ribbon for each strong cover which consists of two ribbons.

For the differential bijection, we note that for $\lambda \in \mathbb{Y}^{i^{\prime}}$, once again, $\mathscr{U}_{\lambda}$ is in natural bijection with the set of $\lambda$-addable corners and $\mathscr{D} u_{\lambda}$ is in natural bijection with the set of $\lambda$-removable corners; in this context the corners are grouped by diagonals of the form $\pm i$ for various $i$. Using the differential bijection in Example 2.9, 


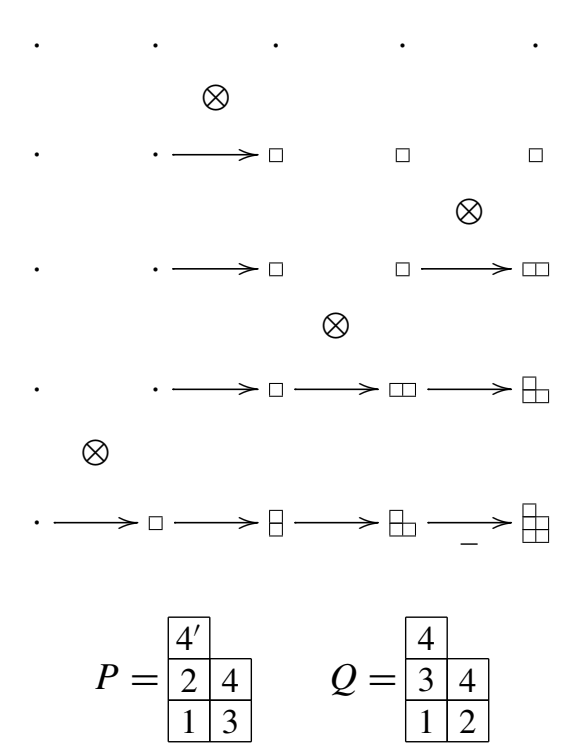

Figure 2. Folded insertion of $\sigma=2431$ for $i^{\prime}=j^{\prime}=1$.

we obtain a folded insertion for the limit $\mathbb{Y}^{i^{\prime}}$ of $\mathscr{C}_{2 n}^{j^{\prime}}$. It defines a bijection from permutations $P_{n}(1)$ to pairs $(P, Q)$ where $P$ and $Q$ are $n$-step strong and weak tableaux with respect to $\mathbb{Y}^{i^{\prime}}$.

For example, we let $i^{\prime}=j^{\prime}=1$ and compute the folded insertion of $\sigma=2431$; see the graph $G$ in Figure 2. For the meaning of $\otimes$ see (9) and Example 3.2. The arrows represent strong covers, and an arrow is labeled with - if the strong cover adds two nonadjacent cells and the marked cell is in a more negative diagonal. The unique arrow labeled with - , corresponds to the entry $4^{\prime}$ in $P$. Strictly speaking we should mark one ribbon for each number used, but when there is no choice we have omitted the marking.

Again, with $i^{\prime}=j^{\prime}=1$ we compute the folded insertion of 4213; see the graph $G$ in Figure 3. Note that there is a unique strong cover that is not a weak cover, corresponding to the domino in $P$ containing $4 \mathrm{~s}$.

5D. Sagan-Worley insertion. We now consider the important case that $i^{\prime}=0$. We must have $j^{\prime}=0$. The following result is straightforward.

Lemma 5.4. The set $\mathscr{C}_{2 n}^{0}$ is the subset of $\mathscr{C}_{2 n}$ of elements fixed by the transpose $\mathrm{tr}$.

Using the fact that $f\left(W_{n}^{0}\right) \subset \widetilde{S}_{2 n}^{0}$, the next result is an easy consequence of Propositions 4.13 and 3.1. A similar statement holds for $i^{\prime}=n$.

Proposition 5.5. Suppose $\lambda \subset \mu$ is a strong cover in $\mathscr{C}_{2 n}^{0} \cong W_{n}^{0}$ with $\operatorname{sc}_{0}^{-1}(\mu)=$ $s c_{0}^{-1}(\lambda) s_{\alpha}$. Then the Chevalley coefficient $\left\langle\alpha^{\vee}, \Lambda_{0}\right\rangle$ is equal to the total number of 


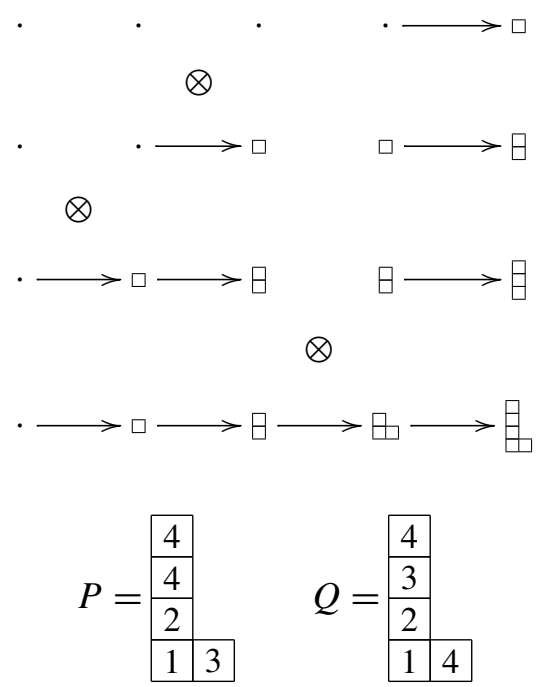

Figure 3. Folded insertion of $\sigma=4213$ for $i^{\prime}=j^{\prime}=1$.

components of $v / \lambda$ for a strong cover $\lambda \subset v$ in $\mathscr{C}_{2 n} \cong \widetilde{S}_{2 n}^{0}$ satisfying $\lambda \subset v \subset \mu$. In particular, if $\lambda \subset \mu$ is also a left weak cover in $\mathscr{C}_{2 n}^{0} \cong W_{n}^{0}$ with $\mu=s_{i} \lambda$ for $i \in I$, then $\left\langle\alpha^{\vee}, \Lambda_{0}\right\rangle$ is equal to the number of $\lambda$-addable corner cells of residue $i$ or $-i$ modulo $2 n$.

Thus for $i^{\prime}=0$, a folded strong tableau of shape $\lambda \in \mathscr{C}_{2 n}^{0}$ is a sequence of strong covers in $\mathscr{C}_{2 n}^{0} \cong W_{n}^{0}$ from $\varnothing$ to $\lambda$, such that every cover has a marked component. A folded weak tableau of shape $\lambda \in \mathscr{C}_{2 n}^{0}$ is a sequence of weak covers in $\mathscr{C}_{2 n}^{0} \cong W_{n}^{0}$ going from $\varnothing$ to $\lambda$; no marking is necessary. With these explicit descriptions we have the following:

Corollary 5.6. LLMS insertion induces a bijection from the set of permutations $P_{n}(1)$ to pairs $(P, Q)$ of tableaux of the same shape $\lambda \in \mathscr{C}_{2 n}^{0}$, where $\ell\left(s c_{0}^{-1}(\lambda)\right)=n$, $P$ is a folded strong tableau and $Q$ a folded weak tableau.

Again we now consider the $n \rightarrow \infty$ limit. In this case the limit of $\mathscr{C}_{2 n}^{0}$ is the set $\bigvee^{0}$ of partitions fixed under the transpose. The strong and weak orders both converge to the same order on $\bigvee^{0}$. Since added cells are in transpose-symmetric positions, when marking a strong cover of the form $\lambda \lessdot s_{i} \lambda$, one must mark either the added cell in diagonal $i$ or $-i$ if $i>0$.

Folded weak tableaux $Q$ are in obvious bijection with standard shifted tableaux $Q^{*}$, given by taking only the part on one side of the diagonal. A similarly obvious 
bijection exists from folded strong tableaux to standard shifted tableaux in which off-diagonal entries may or may not have a mark; we choose the bijection so that a mark in a shifted tableau $P^{*}$ indicates that the corresponding cell with negative diagonal index is marked in the folded strong tableau $P$.

Thus we have the correct kinds of tableaux to compare with Sagan-Worley insertion.

Example 5.7. Let $n=7$ and $\sigma=2673541$. See Example 3.2 for the way to interpret $\sigma$ and the symbols $\otimes$. We draw the graph $G_{i j}$ for the folded insertion of $\sigma$. We draw arrows to represent strong covers and place an asterisk on an edge if the marked cell is on a negative diagonal. $P$ and $Q$ are the folded strong and weak tableaux respectively. $P^{*}$ and $Q^{*}$ are the shifted tableaux corresponding to $P$ and $Q$.

By reformulating Sagan-Worley insertion using Fomin's setup, we obtain the following theorem. Note the exchange of $P^{*}$ and $Q^{*}$.

Theorem 5.8. Let $\sigma$ map to $(P, Q)$ under folded insertion. Then the pair $\left(Q^{*}, P^{*}\right)$ of shifted tableaux, is the image of $\sigma^{-1}$ under Sagan-Worley insertion.

Remark 5.9. In [1989, Proposition 6.2], Haiman relates Sagan-Worley shifted insertion with left-right insertion. It is natural to ask whether one can connect Sections 2E and 4D in a similar manner. Unfortunately, a straightforward generalization of Haiman's result does not appear to be possible. For example when $\pi$ has order 2, one would need to relate the left-right (or mixed) insertion of a colored permutation on $2 r$ letters with folded insertion of a permutation on $r$ letters. Length considerations show that this can be done only if each orbit of $\pi$ on $J$ has order 2, which nearly never happens in our setup.

\section{Distributive parabolic quotients}

6A. Proctor's classification. Let $W$ be a finite irreducible Weyl group with simple generators $\left\{s_{i} \mid i \in I\right\}$ and set of reflections $T$. Recall the notations $W_{J}$ and $W^{J}$ from before Proposition 2.16. We have

$$
W^{J}=\left\{w \in W \mid w<w s_{i} \text { for any } i \in J\right\} .
$$

Proctor [1984] classified the cases when $W^{J}$ is a distributive lattice under the weak order. In all such cases, Stembridge [1996] showed that the weak and strong orders agree on $W^{J}$ and that $W_{J}$ is a maximal parabolic subgroup of $W$, that is, $J=I \backslash\{i\}$ for some $i \in I$. We call such $W^{J}:=W^{i}$ distributive parabolic quotients.

Theorem 6.1 [Proctor 1984]. The distributive parabolic quotients are:

(1) $W \simeq A_{n} ; J=I \backslash\{i\}$ for any $i \in I$. 


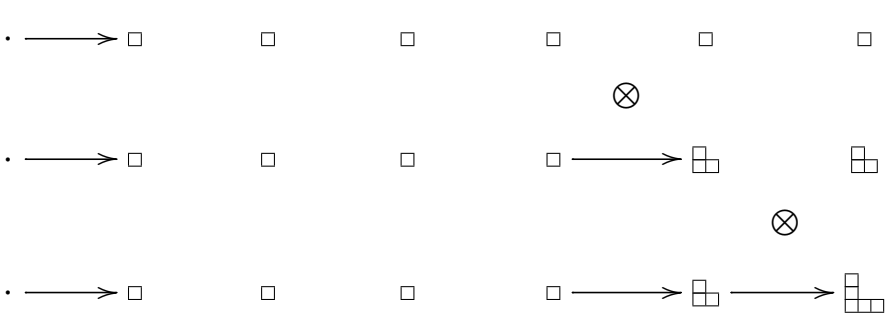

$\otimes$
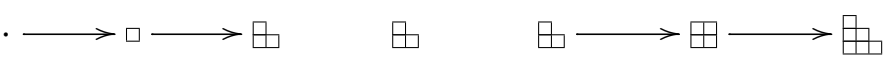

$\otimes$
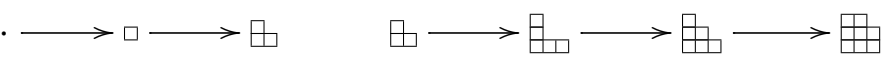

$\otimes$

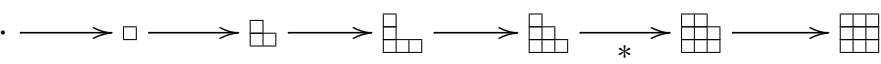

$\otimes$

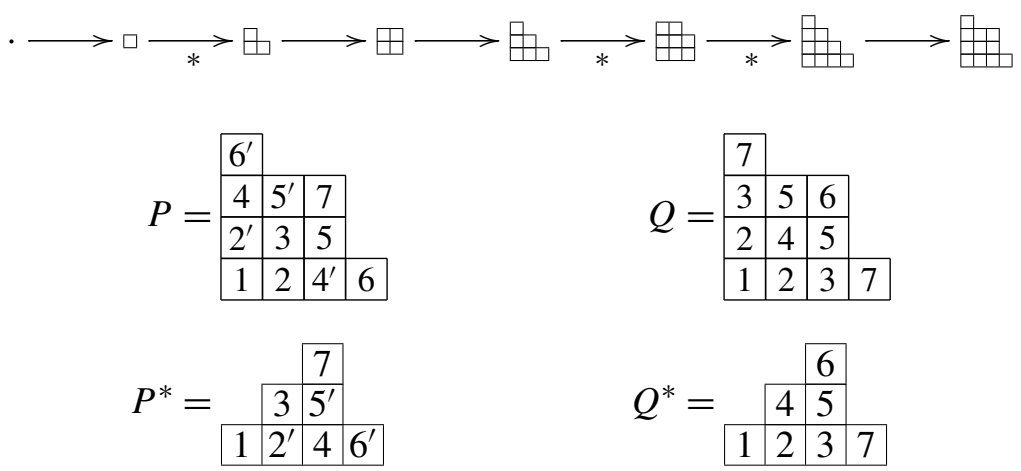

Figure 4. Growth diagram illustrating Theorem 5.8.

(2) $W \simeq B_{n} ; W_{J} \simeq B_{n-1}$ or $W_{J} \simeq A_{n-1}$.

(3) $W \simeq D_{n} ; W_{J} \simeq D_{n-1}$ or $W_{J} \simeq A_{n-1}$.

(4) $W \simeq G_{2} ; J=I \backslash\{i\}$ for any $i \in I$.

In [Stembridge 1996], it is shown that these cases are also exactly the parabolic quotients $W^{J}$ of Weyl groups such that every element $w \in W^{J}$ is fully commutative, that is, every two reduced decompositions of $w$ can be obtained from each other using just the relations of the form $s_{i} s_{j}=s_{j} s_{i}$ for $i, j \in I$. 
6B. Distributive labeled posets. We need a slightly more precise form of the results of Proctor and Stembridge. If $Q$ is a finite poset we let $J(Q)$ denote the poset of (lower) order ideals of $Q$. The poset $J(Q)$ is a distributive lattice and the fundamental theorem of finite distributive posets [Stanley 1999] says that the correspondence $Q \mapsto J(Q)$ is a bijection between finite posets and finite distributive lattices. Suppose $P$ is a finite poset and $\omega:\{x \lessdot y\} \rightarrow A$ is a labeling of the edges of the Hasse diagram of $P$ with elements of some set $A$. We call $(P, \omega)$ an edge-labeled poset. We say that $(P, \omega)$ is a distributively labeled lattice if

(1) $P=J(Q)$ is a distributive lattice; and

(2) there is a vertex (element) labeling $\pi: Q \rightarrow A$ such that

$$
\omega(I \backslash\{q\} \lessdot I)=\pi(q)
$$

for any $I \in J(Q)$ and $q$ maximal in $I$.

If $W$ is a Weyl group, we may label the edges of the Hasse diagram of the weak order $(W, \prec)$ with simple reflections: the cover $w \prec s_{i} w$ is labeled with $s_{i}$. We denote the resulting edge-labeled poset by $W_{\text {weak }}$. Similarly define $W_{\text {strong }}$ to be the strong order where $w \lessdot w t$ is labeled with $t \in T$. These labeled posets restrict to give labeled posets $W_{\text {weak }}^{i}$ and $W_{\text {strong }}^{i}$. Note that each cover relation in $W^{i}$ under either order is itself a cover relation in $W$. Thus $W_{\text {weak }}^{i}$ and $W_{\text {strong }}^{i}$ are induced subgraphs of $W_{\text {weak }}$ and $W_{\text {strong }}$.

Theorem 6.2. Suppose $W^{i}$ is a distributive parabolic quotient. Then the strong and weak orders on $W^{i}$ coincide. In particular $W_{\text {weak }}^{i}$ and $W_{\text {strong }}^{i}$ are distributively labeled lattices.

Stembridge [1996, Theorem 2.2] proved that $W_{\text {weak }}^{i}$ is a distributively labeled lattice. For the sake of completeness we give a self-contained proof of Theorem 6.2.

6C. Cominuscule parabolic quotients. Let $\Phi$ be an irreducible finite root system and $W$ be its Weyl group. Let $\Phi=\Phi^{+} \sqcup \Phi^{-}$denote the decomposition of the roots into the disjoint subsets of positive and negative roots. Let $\theta=\sum_{i \in I} a_{i} \alpha_{i}$ denote the highest root of $\Phi$. We say that $i \in I$ is cominuscule if $a_{i}=1$.

It can be checked case-by-case using Theorem 6.1 that the distributive parabolic quotients $W^{i}$ correspond to cominuscule nodes $i \in I$ except in the cases $W=$ $G_{2}, W^{i}=B_{n} / A_{n-1}$ or $W^{i}=C_{n} / C_{n-1}$. In the latter two cases, one may use the isomorphic quotients given by their duals $C_{n} / A_{n-1}$ and $B_{n} / B_{n-1}$, which are cominuscule.

For now we suppose that a cominuscule node $i \in I$ has been fixed. If $\alpha$ and $\beta$ are two roots, we say $\alpha \geq \beta$ if $\alpha-\beta$ is a sum of positive roots. Recall that $\theta$ is the unique maximal root under this order. Let $\Phi^{(i)}$ denote the poset of positive roots 
which lie above $\alpha_{i}$. Clearly $\theta \in \Phi^{(i)}$. The inversion set of $w \in W$ is defined by

$$
\operatorname{Inv}(w)=\left\{\alpha \in \Phi^{+} \mid w \alpha<0\right\} .
$$

Lemma 6.3. Suppose $\alpha, \beta \in \Phi^{(i)}$. Write $s_{\alpha} \beta=\beta+k \alpha$ where $k=-\left\langle\alpha^{\vee}, \beta\right\rangle$. Then $k \in\{0,-1,-2\}$ and the following facts hold:

(1) If $\alpha$ and $\beta$ are incomparable then $s_{\alpha} \beta=\beta$.

(2) If $\alpha>\beta$ then $s_{\alpha} \beta$ is equal to one of the following: (i) $\beta$; (ii) $-\gamma$ where $\gamma \in \Phi^{+} \backslash \Phi^{(i)}$; or (iii) $-\gamma$ where $\gamma>\alpha$.

Proof. To obtain the bounds on $k$ we observe that for all roots $\gamma \in \Phi,-\theta \leq \gamma \leq \theta$, so that the coefficient of $\alpha_{i}$ in $\gamma$, lies between the corresponding coefficients in $-\theta$ and $\theta$, which are -1 and 1 by the assumption that $i$ is cominuscule.

Suppose that $\alpha$ and $\beta$ are incomparable. Then $\beta-\alpha$ is neither positive nor negative and hence not a root. Since the roots in $\Phi$ occur in strings, we must have $k=0$.

If $\alpha>\beta$, the three cases correspond to $k=0, k=-1$, and $k=-2$.

For our results on distributive parabolic quotients, we require the following result, which is a slight strengthening of [Thomas and Yong 2006, Proposition 2.1, Lemma 2.2]. We include a self-contained proof, part of which is the same as the proof of [Thomas and Yong 2006, Proposition 2.1]. In particular we prove directly that the edge labeled poset $W_{\text {strong }}^{i}$ defined in Section 6B is a distributively labeled lattice.

Proposition 6.4. The map $w \longmapsto \operatorname{Inv}(w)$ defines an isomorphism of posets $\left.\operatorname{Inv}\right|_{W^{i}}$ : $\left(W^{i}, \leq\right) \rightarrow J\left(\Phi^{(i)}\right)$. Moreover, if $u \lessdot w$ for $u, w \in W^{i}$, then writing $w=u s_{\alpha}$ for $\alpha \in \Phi^{+}$, we have $\alpha \in \Phi^{(i)}$ and $\operatorname{Inv}(w)=\operatorname{Inv}(u) \sqcup\{\alpha\}$.

Proof. Let $w \in W^{i}$. First we show that $\operatorname{Inv}(w) \subset \Phi^{(i)}$. Suppose that $\gamma \in \operatorname{Inv}(w) \backslash$ $\Phi^{(i)}$. If $\gamma=\alpha_{k}$ where $k \neq i$ this means $w s_{k}<w$ which contradicts the assumption that $w \in W^{i}$. Otherwise $\gamma=\delta+\rho$ where $\delta, \rho \in \Phi^{+} \backslash \Phi^{(i)}$. Since $w \gamma<0$ we have $w \delta<0$ or $w \rho<0$ so the same argument applies. Repeating we obtain a contradiction.

Now we show that $\operatorname{Inv}(w) \in J\left(\Phi^{(i)}\right)$. Suppose $\alpha \in \operatorname{Inv}(w)$ and $\beta<\alpha$. Then $\gamma=\alpha-\beta \in \Phi^{+} \backslash \Phi^{(i)}$ since the coefficient of $\alpha_{i}$ in $\gamma$ is zero. Since $\operatorname{Inv}(w) \subset \Phi^{(i)}$, we have $\gamma \notin \operatorname{Inv}(w)$, that is, $w \alpha-w \beta=w \gamma>0$. Since $w \alpha<0$ this shows that $w \beta<0$ as desired. Thus Inv $\left.\right|_{W^{i}}$ is well-defined.

Next we show that Inv $\left.\right|_{W^{i}}$ sends covers to covers. Let $u \lessdot w$ with $u, w \in W^{i}$ and $\alpha \in \Phi^{+}$such that $w=u s_{\alpha}$. Then $0>w \alpha=-u \alpha$ so $\alpha \in \operatorname{Inv}(w) \backslash \operatorname{Inv}(u)$. For all $\beta \in \operatorname{Inv}(u)$, since $\operatorname{Inv}(u) \in J\left(\Phi^{(i)}\right), \alpha>\beta$ or $\alpha>\beta$. Either way we have $w \beta=u s_{\alpha} \beta<0$, since by Lemma $6.3, s_{\alpha} \beta$ is either equal to $\beta$ or $-\gamma$ for 
$\gamma \in \Phi^{+} \backslash \operatorname{Inv}(u)$. That is, $\operatorname{Inv}(u) \subset \operatorname{Inv}(w)$. Since $|\operatorname{Inv}(w)|=|\operatorname{Inv}(u)|+1$ it follows that $\operatorname{Inv}(w)=\operatorname{Inv}(u) \sqcup\{\alpha\}$, so that $\operatorname{Inv}(u) \subset \operatorname{Inv}(w)$ is a covering relation in $J\left(\Phi^{(i)}\right)$.

Next we show that every covering relation in $J\left(\Phi^{(i)}\right)$ is the image of a covering relation in $W^{i}$, and in particular, that $\left.\mathrm{Inv}\right|_{W^{i}}$ is onto. An arbitrary covering relation in $J\left(\Phi^{(i)}\right)$ is given by $S \backslash\{\alpha\} \subset S$ where $S \in J\left(\Phi^{(i)}\right)$ and $\alpha$ is maximal in $S$.

By induction there is a $u \in W^{i} \operatorname{such}$ that $\operatorname{Inv}(u)=S \backslash\{\alpha\}$. Let $w=u s_{\alpha}$. It suffices to show that

$$
\operatorname{Inv}(w)=S \quad \text { and } \quad w \in W^{i} .
$$

The second claim follows from the first since none of the $\alpha_{k}$ for $k \neq i$ lie in $\operatorname{Inv}(w)$. For the first claim, since $\alpha \in \Phi^{(i)} \backslash \operatorname{Inv}(u)$, we may argue as before to show that $S=\operatorname{Inv}(u) \sqcup\{\alpha\} \subset \operatorname{Inv}(w)$.

For the opposite inclusion, suppose $\beta \in \Phi^{+} \backslash S$. We must show that $w \beta>0$. Write $s_{\alpha} \beta=\beta+k \alpha$ for $k \in \mathbb{Z}$. If $k=0$ then we are done as before. If $k>0$ then $s_{\alpha} \beta>\alpha$, so that $s_{\alpha} \beta \in \Phi^{+} \backslash S$ since $S$ is an order ideal. But then $s_{\alpha} \beta \notin \operatorname{Inv}(u)$ so $w \beta>0$. So we may assume that $k<0$.

Suppose first that $\beta \in \Phi^{(i)}$. We may assume that $\alpha$ and $\beta$ are comparable by Lemma 6.3. Since $S$ is an order ideal we have $\beta>\alpha$. If $k=-1$ then $s_{\alpha} \beta=\beta-\alpha \in$ $\Phi^{+} \backslash \Phi^{(i)}$ since the coefficient of $\alpha_{i}$ is 1 in both $\alpha$ and $\beta$. In particular $s_{\alpha} \beta \notin \operatorname{Inv}(u)$ so $w \beta>0$. Otherwise $k=-2$. Then $s_{\alpha} \beta=\beta-2 \alpha<0$. We have $0<\beta-\alpha<\alpha$ and $-s_{\alpha} \beta=2 \alpha-\beta=\alpha-(\beta-\alpha)<\alpha$. Since $S$ is an order ideal it follows that $-s_{\alpha} \beta \in \operatorname{Inv}(u)$ and $w \beta=u s_{\alpha} \beta>0$ as desired.

Otherwise $\beta \in \Phi^{+} \backslash \Phi^{(i)}$. Since $i$ is cominuscule we have $k \in\{-1,0,1\}$. We assume $k=-1$ as the other cases were already done. Then $s_{\alpha} \beta=\beta-\alpha<0$ since its coefficient of $\alpha_{i}$ is -1 . Moreover $\alpha-\beta \in \Phi^{(i)}$. Since $\alpha>\alpha-\beta$ and $S$ is an order ideal, it follows that $\alpha-\beta \in \operatorname{Inv}(u)$. Therefore $w \beta=u s_{\alpha} \beta>0$ as desired.

We have shown that every cover in $J\left(\Phi^{(i)}\right)$ is the image under $\left.\operatorname{Inv}\right|_{W^{i}}$ of a cover in $\left(W^{i}, \leq\right)$.

The bijectivity of Inv| $\left.\right|_{W^{i}}$ follows by induction and the explicit description of the image of a cover under Inv $\left.\right|_{W^{i}}$.

Proof of Theorem 6.2. For the case $W=G_{2}$, both labeled posets $W_{\text {weak }}^{i}$ and $W_{\text {strong }}^{i}$ are chains, so the result follows immediately. Thus we may assume that $W^{i}$ is a cominuscule parabolic quotient.

For $W_{\text {strong }}^{i}$ the result follows from Proposition 6.4. We label the vertices of $\Phi^{(i)}$ by reflections, defining $\pi: \Phi^{(i)} \rightarrow T$ by $\pi(\alpha)=s_{\alpha}$. Each cover $w \lessdot w s_{\alpha}$ in $W_{\text {strong }}^{i}$ corresponds to adding $\alpha \in \Phi^{(i)}$ to $\operatorname{Inv}(w)$. Thus the edge label of $w \lessdot w s_{\alpha}$ agrees with the vertex label $\pi(\alpha)=s_{\alpha}$.

For the weak order $W_{\text {weak }}^{i}$ let us consider two covers $w \lessdot w s_{\alpha}=s_{\beta} w$ and $v \lessdot v s_{\alpha}=$

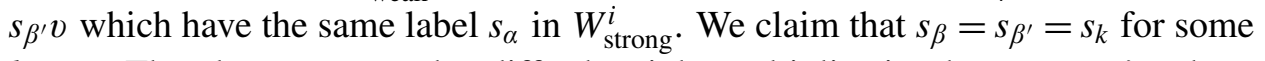
$k \in I$. The elements $w$ and $v$ differ by right multiplication by some $s_{\gamma}$ 's where 
$\gamma \in \Phi^{(i)}$ is incomparable with $\alpha$; this is accomplished by passing between $w$ or $v$ to the element $u \in W^{i}$ such that $\operatorname{Inv}(u)=\operatorname{Inv}(w) \cap \operatorname{Inv}(v)$. By Lemma 6.3 these $s_{\gamma}$ 's commute with $s_{\alpha}$, and so $w \alpha=v \alpha$. This gives us a map $f: \Phi^{(i)} \rightarrow \Phi^{+}$defined by $f(\alpha)=\beta=w \alpha$, which does not depend on $w \in W^{i}$ as long as $w \lessdot w s_{\alpha}$.

To show that $f(\alpha)$ is simple for each $\alpha \in \Phi^{(i)}$, consider a reduced word $w s_{\alpha}=$ $s_{k_{1}} s_{k_{2}} \cdots s_{k_{l}}$. We know that $w^{(r)}=s_{k_{r}} \cdots s_{k_{l}} \in W^{i}$ and that $\operatorname{Inv}\left(w^{(r)}\right)$ differs from $\operatorname{Inv}\left(w^{(r+1)}\right)$ by some root in $\Phi^{(i)}$ since $w^{(r+1)} \lessdot w^{(r)}$. For some value $r=r^{*}$, this root is $\alpha$ and by the well-definedness just proved $f(\alpha)=\alpha_{k_{r^{*}}}$, since $w^{\left(r^{*}\right)}=$ $w^{\left(r^{*}+1\right)} s_{\alpha}$. This shows that the strong order and weak order on $W^{i}$ coincide, and that $W_{\text {weak }}^{i}$ is isomorphic to the poset of order ideals of $\Phi^{(i)}$ where $\Phi^{(i)}$ is labeled with $\pi(\alpha)=f(\alpha)$.

\section{Distributive subgraphs of Kac-Moody graded graphs}

In this section we apply Theorem 6.2 to the dual graded graphs constructed in Section 2.

Let $\mathfrak{g}=\mathfrak{g}(A)$ be the Kac-Moody algebra associated to the generalized Cartan matrix $A$ and let $W$ be its Weyl group. Let $W_{\text {fin }} \subset W$ be a finite parabolic subgroup corresponding to some index set $I^{\prime} \subset I$. Now suppose that $W_{\text {fin }}$ has a distributive parabolic quotient as in Theorem 6.1 corresponding to $J=I^{\prime} \backslash\{i\} \subset I^{\prime}$. We let $W^{J} \subset W_{\text {fin }}$ denote the distributive parabolic quotient (we use $W^{J}$ instead of $W^{i}$ in this section since $W^{J}$ is not a maximal parabolic quotient of $W$, but of $W_{\text {fin }}$ ).

Now let $(\Lambda, K) \in P^{+} \times Z^{+}$and $\left(\Gamma_{s}(\Lambda), \Gamma_{w}(K)\right)$ be the pair of dual graded graphs constructed in Section 2. By restricting to the set of vertices $W^{J} \subset W_{\text {fin }} \subset$ $W$ we obtain the induced pair of graded graphs $\left(\Gamma_{s}(\Lambda), \Gamma_{w}(K)\right)^{J}$. These graded graphs are not dual (see Remark 2.1) but they still have rich combinatorics.

The distributive lattice $\left(W^{J}, \leq\right)$ has two edge labelings. Recall that in $W_{\text {strong }}^{J}$, the edge $v \lessdot w=v s_{\alpha}$ is labeled either by the reflection $s_{\alpha}$, while in the strong KacMoody subgraph $\Gamma_{s}^{J}(\Lambda)$, the edge $v \lessdot w=v s_{\alpha}$ is labeled by the integer $\left\langle\alpha^{\vee}, \Lambda\right\rangle$. Similarly the distributive lattice $\left(W^{J}, \preceq\right)$ has two edge labelings; in $W_{\text {weak }}^{J}$, the edge $v \prec s_{j} v$ is labeled by the simple reflection $s_{j}$, while in the weak Kac-Moody subgraph $\Gamma_{w}^{J}(K)$, the edge $v \prec s_{j} v$ is labeled by the integer $\left\langle K, \Lambda_{j}\right\rangle$. The following result is an immediate consequence of Theorem 6.2.

Theorem 7.1. The induced graded subgraphs $\Gamma_{s}^{J}(\Lambda)$ and $\Gamma_{w}^{J}(K)$ are distributively labeled lattices.

Thus $\Gamma_{s}^{J}(\Lambda)\left(\operatorname{resp} . \Gamma_{w}^{J}(K)\right)$ can be thought of as the poset of order ideals in some integer labeled poset $P^{J}$ (resp. $Q^{J}$ ). The $\Lambda$-strong and $K$-weak tableaux can be thought of as linear extensions of $P^{J}$ and $Q^{J}$ with additional markings.

In the rest of the paper, we give examples of the posets $P^{J}$ and $Q^{J}$ and relate them to classically understood tableaux. In each case we let $\mathfrak{g}$ be of untwisted affine 


\begin{tabular}{|c|c|}
\hline Root system & Dynkin Diagram \\
\hline$A_{n}$ & $\begin{array}{cccccc}\circ & 0 & 0 & \bullet & 0 & 0 \\
1 & 2 & \cdots & i & \cdots & n\end{array}$ \\
\hline$C_{n}, n \geq 3$ & $\begin{array}{llllll}0 & 0 & 0 & 0 & 0< \\
1 & 2 & \cdots & \cdots & n\end{array}$ \\
\hline$D_{n}, n \geq 4$ & $\begin{array}{lllll}\circ & 0 & 0 & \circ & \circ \\
1 & 2 & \cdots & \cdots & \cdots\end{array}$ \\
\hline$E_{6}$ & 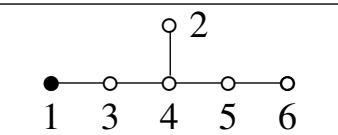 \\
\hline$E_{7}$ & 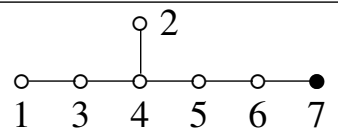 \\
\hline
\end{tabular}

Figure 5. Some cominuscule parabolic quotients.

type, $I_{\text {fin }}=I \backslash\{0\}$ and $J=I \backslash\{i\}$ for a fixed node $i \in I_{\text {fin }}$ to be specified. We use the canonical central element $K_{\text {can }}=\sum_{i \in I} a_{i}^{\vee} \alpha_{i}^{\vee}$ for $K$ and $\Lambda_{i}$ for the dominant weight. In this case $P^{J}$ and $Q^{J}$ are both labelings of the poset $\Phi^{(i)} \subset \Phi^{+}$for the simple Lie algebra $\mathfrak{g}_{\text {fin }}$ whose Dynkin diagram is the subdiagram of that of $\mathfrak{g}$ given by removing the 0 node. These examples, with the exception of $G_{2}$, can be viewed as providing some additional data for the posets $\Phi^{(i)}$, whose unlabeled versions were given explicitly in [Thomas and Yong 2006]. As in that reference, we rotate the labeled Hasse diagrams clockwise by 45 degrees so that the minimal element is in the southwest corner. In the following, we let $V_{\text {weak }}^{J}, V_{\text {strong }}^{J}$ denote the vertex-labeled posets such that $W_{\text {weak }}^{J}=J\left(V_{\text {weak }}^{J}\right)$ and $W_{\text {strong }}^{J}=J\left(V_{\text {strong }}^{J}\right)$.

7A. Type $\boldsymbol{A}_{\boldsymbol{n}}^{(\mathbf{1})}$. Let $i \in I_{\mathrm{fin}}$ be arbitrary. The poset $\Phi^{(i)}$ consists of elements $\alpha_{p, q}=$ $\alpha_{p}+\cdots+\alpha_{q}$ for $1 \leq p \leq i \leq q \leq n$. The weak labeling of $\Phi^{(i)}$ is given by $\alpha_{p, q} \mapsto s_{p+q-i}$. For example, for $n=7$ and $i=3$ and abbreviating $\alpha_{p, q}$ by $p q$ and $s_{j}$ by $j$, the labelings of $\Phi^{(i)}$ by positive roots and simple reflections are given by

$$
V_{\text {strong }}^{J}=\begin{array}{|l|l|l|l|l|}
13 & 14 & 15 & 16 & 17 \\
\hline 23 & 24 & 25 & 26 & 27 \\
\hline 33 & 34 & 35 & 36 & 37
\end{array} \quad V_{\text {weak }}^{J}=\begin{array}{|l|l|l|l|l|}
\hline 1 & 2 & 3 & 4 & 5 \\
\hline 2 & 3 & 4 & 5 & 6 \\
\hline 3 & 4 & 5 & 6 & 7 \\
\hline
\end{array}
$$

All labelings in $P^{J}$ and $Q^{J}$ are given by the constant 1 . The resulting strong and weak tableaux are usual standard tableaux.

7B. Type $\boldsymbol{C}_{\boldsymbol{n}}^{(\mathbf{1})}$. Let $i=n$. Let $\alpha_{i}=e_{i}-e_{i+1}$ for $1 \leq i \leq n-1$ and $\alpha_{n}=2 e_{n}$ where $e_{i}$ is the $i$-th standard basis element of the weight lattice $\mathbb{Z}^{n}$. Then $\Phi^{(n)}$ consists of 
the roots $\alpha_{i, j}=e_{i}+e_{j}$ for $1 \leq i \leq j \leq n$. We have $a_{i}^{\vee}=1$ for all $i$. For $n=4$ we have

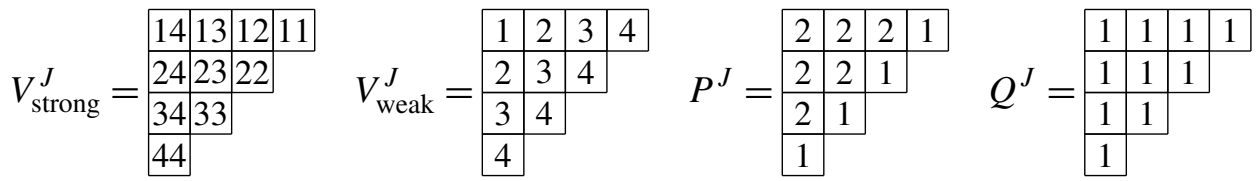

The strong tableaux are shifted standard tableaux with two kinds of markings on off-diagonal entries; these are the standard recording tableaux for shifted insertion [Sagan 1987]. The weak tableaux are standard shifted tableaux.

7C. Type $\boldsymbol{D}_{\boldsymbol{n}}^{(\mathbf{1})}$. Let $i=n$. Letting $\alpha_{i}=e_{i}-e_{i+1}$ for $1 \leq i \leq n-1$ and $\alpha_{n}=e_{n-1}+e_{n}$, the roots of $\Phi^{(n)}$ are given by $\alpha_{p, q}=e_{p}+e_{q}$ for $1 \leq p<q \leq n$. We have $a_{j}^{\vee}=1$ for $j \in\{0,1, n-1, n\}$ and $a_{j}^{\vee}=2$ otherwise. For $n=5$ we give the labelings of $\Phi^{(n)}$ below. Note the 1 in the upper left corner of $Q^{J}$.

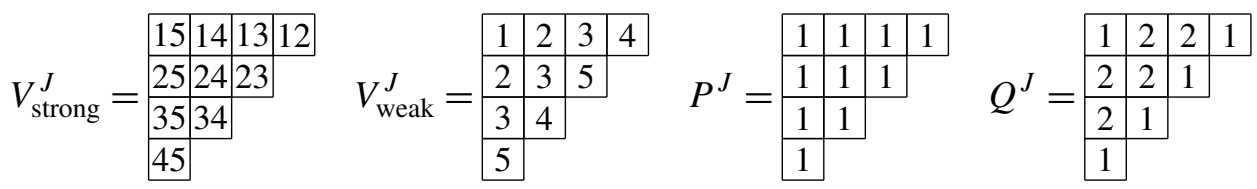

7D. Type E. The computations in this section were made using Stembridge's Coxeter/Weyl package [Stembridge 2004]. In both of the following cases, $P^{J}$ has all labels 1.

For $E_{6}^{(1)}$ and $i=1$ with the Dynkin labeling in Figure 5, we have

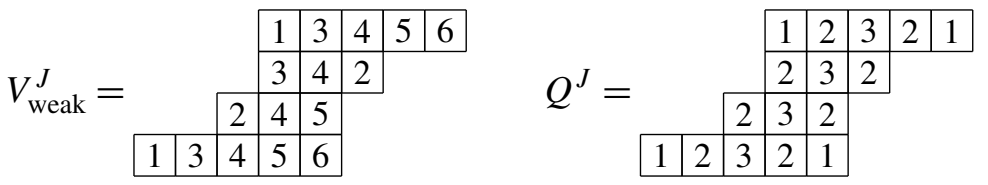

For $E_{7}^{(1)}$ and $i=7$ with the Dynkin labeling in Figure 5, we have

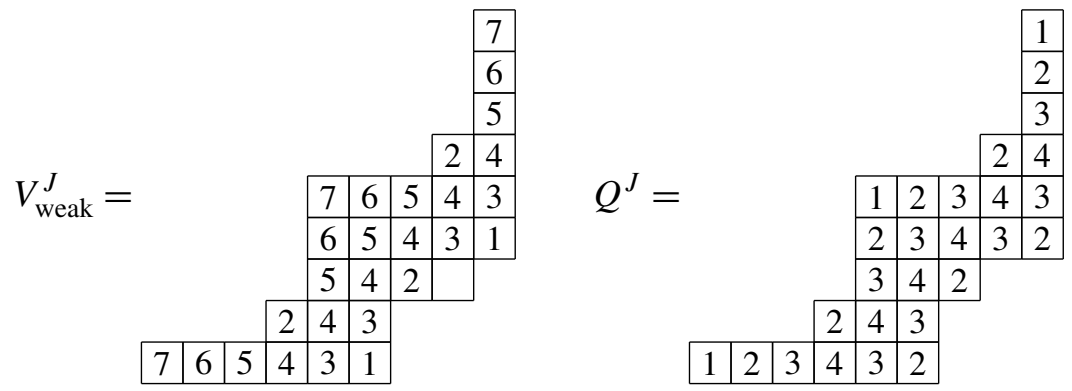

7E. Type $\boldsymbol{G}_{\mathbf{2}}^{(\mathbf{1})}$. This case does not correspond to a cominiscule root. Pick $i=1$ and let $\alpha_{1}, \alpha_{2}$ be the two simple roots, so that the highest root is $3 \alpha_{1}+2 \alpha_{2}$. Then 
$a_{1}^{\vee}=1$ and $a_{2}^{\vee}=2$. Abbreviating the reflection $s_{p \alpha_{1}+q \alpha_{2}}$ by $p q$, we have:

$$
\begin{aligned}
& V_{\text {strong }}^{J}=\begin{array}{l|l|l|l|l|l|l|l|l|l|l|}
1 & 31 & 21 & 32 & 11 \\
\hline
\end{array} \quad P^{J}=\begin{array}{ll|l|l|l|l|l|}
1 & 3 & 2 & 3 & 1 \\
\hline
\end{array} \\
& V_{\text {weak }}^{J}=\begin{array}{l|l|l|l|l|}
1 & 2 & 1 & 2 & 1
\end{array} \quad Q^{J}=\begin{array}{ll|l|l|l|l|}
1 & 2 & 1 & 2 & 1 \\
\hline
\end{array}
\end{aligned}
$$

\section{References}

[Deodhar 1977] V. V. Deodhar, "Some characterizations of Bruhat ordering on a Coxeter group and determination of the relative Möbius function”, Invent. Math. 39:2 (1977), 187-198. MR 55 \#8209 Zbl 0346.20032

[Fomin 1986] S. V. Fomin, "The generalized Robinson-Schensted-Knuth correspondence", Zap. Nauchn. Sem. Leningrad. Otdel. Mat. Inst. Steklov. (LOMI) 155:Differentsialnaya Geometriya, Gruppy Li i Mekh. VIII (1986), 156-175, 195. MR 88b:06003 Zbl 0661.05004

[Fomin 1994] S. Fomin, "Duality of graded graphs", J. Algebraic Combin. 3:4 (1994), 357-404. MR 95i:05088 Zbl 0810.05005

[Fomin 1995] S. Fomin, "Schensted algorithms for dual graded graphs", J. Algebraic Combin. 4:1 (1995), 5-45. MR 95m:05246 Zbl 0817.05077

[Haiman 1989] M. D. Haiman, “On mixed insertion, symmetry, and shifted Young tableaux”, $J$. Combin. Theory Ser. A 50:2 (1989), 196-225. MR 90j:05014 Zbl 0697.05005

[Humphreys 1990] J. E. Humphreys, Reflection groups and Coxeter groups, Cambridge Studies in Advanced Mathematics 29, Cambridge University Press, Cambridge, 1990. MR 92h:20002 Zbl 0725.20028

[Kac 1990] V. G. Kac, Infinite-dimensional Lie algebras, Third ed., Cambridge University Press, Cambridge, 1990. MR 92k:17038 Zbl 0716.17022

[Kostant and Kumar 1986] B. Kostant and S. Kumar, "The nil Hecke ring and cohomology of $G / P$ for a Kac-Moody group G”, Adv. in Math. 62:3 (1986), 187-237. MR 88b:17025b Zbl 0641.17008

[Lam 2006] T. Lam, "Schubert polynomials for the affine Grassmannian", to appear in J. Amer. Math. Soc. (2006). math.CO/0603125

[Lam and Shimozono $\geq 2007]$ T. Lam and M. Shimozono, "Dual graded graphs arising from combinatorial Hopf algebras". in preparation.

[Lam et al. 2006] T. Lam, L. Lapointe, J. Morse, and M. Shimozono, “Affine insertion and Pieri rules for the affine Grassmannian”, preprint, 2006. math.CO/0609110

[Lusztig 1993] G. Lusztig, Introduction to quantum groups, Progress in Mathematics 110, Birkhäuser, Boston, 1993. MR 94m:17016 Zbl 0788.17010

[Misra and Miwa 1990] K. Misra and T. Miwa, "Crystal base for the basic representation of $U_{q}(\mathfrak{s l}(n)) ”$, Comm. Math. Phys. 134:1 (1990), 79-88. MR 91j:17021 Zbl 0724.17010

[Nanba 2005] M. Nanba, "Bruhat order on the fixed-point subgroup by a Coxeter graph automorphism”, J. Algebra 285:2 (2005), 470-480. MR 2005k:20095 Zbl 1069.20027

[Proctor 1984] R. A. Proctor, "Bruhat lattices, plane partition generating functions, and minuscule representations”, European J. Combin. 5:4 (1984), 331-350. MR 86h:17007 Zbl 0562.05003

[Proctor 1999] R. A. Proctor, "Minuscule elements of Weyl groups, the numbers game, and $d$ complete posets", J. Algebra 213:1 (1999), 272-303. MR 2000a:05219 Zbl 0969.05068

[Sagan 1987] B. E. Sagan, "Shifted tableaux, Schur $Q$-functions, and a conjecture of R. Stanley", $J$. Combin. Theory Ser. A 45:1 (1987), 62-103. MR 88f:05011 Zbl 0661.05010 
[Schensted 1961] C. Schensted, "Longest increasing and decreasing subsequences", Canad. J. Math. 13 (1961), 179-191. MR 22 \#12047 Zbl 0097.25202

[Stanley 1988] R. P. Stanley, "Differential posets", J. Amer. Math. Soc. 1:4 (1988), 919-961. MR 89h:06005 Zbl 0658.05006

[Stanley 1999] R. P. Stanley, Enumerative combinatorics. Vol. 2, Cambridge Studies in Advanced Mathematics 62, Cambridge University Press, Cambridge, 1999. With a foreword by Gian-Carlo Rota and appendix 1 by Sergey Fomin. MR 2000k:05026 Zbl 0928.05001

[Steinberg 1968] R. Steinberg, Endomorphisms of linear algebraic groups, Memoirs of the American Mathematical Society, No. 80, American Mathematical Society, Providence, R.I., 1968. MR 37 \#6288 Zbl 0164.02902

[Stembridge 1996] J. R. Stembridge, "On the fully commutative elements of Coxeter groups", $J$. Algebraic Combin. 5:4 (1996), 353-385. MR 97g:20046 Zbl 0864.20025

[Stembridge 2004] J. Stembridge, Coxeter and Weyl (Maple packages), version 2.4, 2004, Available at http://www.math.Isa.umich.edu/ jrs/maple.html\#coxeter.

[Thomas and Yong 2006] H. Thomas and A. Yong, "A combinatorial rule for (co)minuscule Schubert calculus", preprint, 2006. math.AG/0608276

[Worley 1984] D. Worley, A theory of shifted Young tableaux, Ph.D. thesis, MIT, 1984.

Communicated by Andrei Zelevinsky

Received 2007-03-28 Revised 2007-08-04 Accepted 2007-09-01

tfylam@math.harvard.edu Department of Mathematics, Harvard University, Cambridge MA 02138, USA

http://www.math.harvard.edu/ tfylam/

mshimo@vt.edu

Department of Mathematics, Virginia Polytechnic Institute and State University, Blacksburg, VA 24061-0123, USA 


\section{Algebra \& Number Theory}

www.jant.org

\section{EDITORS}

\section{MANAGING EDITOR}

Bjorn Poonen

University of California

Berkeley, USA

\author{
EDITORIAL BOARD CHAIR \\ David Eisenbud \\ University of California \\ Berkeley, USA
}

\section{BOARD OF EDITORS}

Georgia Benkart

Dave Benson

Richard E. Borcherds

John H. Coates

J-L. Colliot-Thélène

Brian D. Conrad

Hélène Esnault

Hubert Flenner

Edward Frenkel

Andrew Granville

Joseph Gubeladze

Ehud Hrushovski

Craig Huneke

Mikhail Kapranov

Yujiro Kawamata

János Kollár

Hendrik W. Lenstra

Yuri Manin

Barry Mazur
University of Wisconsin, Madison, USA

University of Aberdeen, Scotland

University of California, Berkeley, USA

University of Cambridge, UK

CNRS, Université Paris-Sud, France

University of Michigan, USA

Universität Duisburg-Essen, Germany

Ruhr-Universität, Germany

University of California, Berkeley, USA

Université de Montréal, Canada

San Francisco State University, USA

Hebrew University, Israel

University of Kansas, USA

Yale University, USA

University of Tokyo, Japan

Princeton University, USA

Universiteit Leiden, The Netherlands

Northwestern University, USA

Harvard University, USA
Susan Montgomery

Shigefumi Mori

Andrei Okounkov

Raman Parimala

Victor Reiner

Karl Rubin

Peter Sarnak

Michael Singer

Ronald Solomon

Vasudevan Srinivas

J. Toby Stafford

Bernd Sturmfels

Richard Taylor

Ravi Vakil

Michel van den Bergh

Marie-France Vignéras

Kei-Ichi Watanabe

Andrei Zelevinsky

Efim Zelmanov
University of Southern California, USA

RIMS, Kyoto University, Japan

Princeton University, USA

Emory University, USA

University of Minnesota, USA

University of California, Irvine, USA

Princeton University, USA

North Carolina State University, USA

Ohio State University, USA

Tata Inst. of Fund. Research, India

University of Michigan, USA

University of California, Berkeley, USA

Harvard University, USA

Stanford University, USA

Hasselt University, Belgium

Université Paris VII, France

Nihon University, Japan

Northeastern University, USA

University of California, San Diego, USA

\section{PRODUCTION}

ant@mathscipub.org

Paulo Ney de Souza, Production Manager

Silvio Levy, Senior Production Editor

See inside back cover or www.jant.org for submission instructions.

The subscription price for 2007 is US \$120/year for the electronic version, and \$180/year for print and electronic. Subscriptions, requests for back issues from the last three years and changes of subscribers address should be sent to Mathematical Sciences Publishers, Department of Mathematics, University of California, Berkeley, CA 94720-3840, USA.

Algebra \& Number Theory (ISSN 1937-0652) at Mathematical Sciences Publishers, Department of Mathematics, University of California, Berkeley, CA 94720-3840 is published continuously online. Periodical rate postage paid at Berkeley, CA 94704, and additional mailing offices.

ANT peer-review and production is managed by EditFLOW ${ }^{\mathrm{TM}}$ from Mathematical Sciences Publishers.

PUBLISHED BY

mathematical sciences publishers

http://www.mathscipub.org

A NON-PROFIT CORPORATION

Typeset in LATEX

Copyright $@ 2007$ by Mathematical Sciences Publishers 


\section{Algebra \& Number Theory}

Volume $1 \quad$ No. $4 \quad 2007$

Finite descent obstructions and rational points on curves

MiCHAEL STOLL

Del Pezzo surfaces and representation theory

Vera V. Serganova and Alexei N. Skorobogatov

The zeta function of monomial deformations of Fermat hypersurfaces

REMKE KLOOSTERMAN

THOMAS F. LAM and MARK SHIMOZONO 